\title{
PIM-2 protein kinase negatively regulates $T$ cell responses in transplantation and tumor immunity
}

\author{
Anusara Daenthanasanmak, ${ }^{1}$ Yongxia Wu, ${ }^{1}$ Supinya lamsawat, ${ }^{1}$ Hung D. Nguyen, ${ }^{1}$ David Bastian, ${ }^{1}$ MengMeng Zhang, ${ }^{1}$ \\ M. Hanief Sofi, ${ }^{1}$ Shilpak Chatterjee, ${ }^{2}$ Elizabeth G. Hill, ${ }^{3}$ Shikhar Mehrotra, ${ }^{2}$ Andrew S. Kraft, ${ }^{4}$ and Xue-Zhong Yu ${ }^{1,5}$ \\ 'Department of Microbiology and Immunology, ${ }^{2}$ Department of Surgery, and ${ }^{3}$ Department of Public Health Science, Hollings Cancer Center, Medical University of South Carolina, Charleston, \\ South Carolina, USA. ${ }^{4}$ University of Arizona Cancer Center, Tucson, Arizona, USA. ${ }^{5}$ Department of Medicine, Medical University of South Carolina, Charleston, South Carolina, USA.
}

\begin{abstract}
PIM kinase family members play a crucial role in promoting cell survival and proliferation via phosphorylation of their target substrates. In this study, we investigated the role of the PIM kinases with respect to T cell responses in transplantation and tumor immunity. We found that the PIM-2 isoform negatively regulated T cell responses to alloantigen, in contrast to the PIM-1 and PIM-3 isoforms, which acted as positive regulators. T cells deficient in PIM-2 demonstrated increased T cell differentiation toward Th1 subset, proliferation, and migration to target organs after allogeneic bone marrow transplantation, resulting in dramatically accelerated graft-versus-host disease (CVHD) severity. Restoration of PIM-2 expression markedly attenuated the pathogenicity of PIM-2-deficient T cells to induce CVHD. On the other hand, mice deficient in PIM-2 readily rejected syngeneic tumor, which was primarily dependent on CD8 ${ }^{+} \mathrm{T}$ cells. Furthermore, silencing PIM-2 in polyclonal or antigen-specific CD8 ${ }^{+} \mathrm{T}$ cells substantially enhanced their antitumor response in adoptive $\mathrm{T}$ cell immunotherapy. We conclude that PIM-2 kinase plays a prominent role in suppressing $\mathrm{T}$ cell responses, and provide a strong rationale to target PIM-2 for cancer immunotherapy.
\end{abstract}

\section{Introduction}

The provirus integration sites for Moloney murine leukemia virus (PIM) kinases are a highly conserved family of serine/threonine kinases $(1,2)$. The PIM kinase family is composed of 3 different isoforms, PIM-1, PIM-2, and PIM-3, which share high homology at the amino acid sequences (3) and have functional redundancy (4). PIM-2 kinase is expressed in hematopoietic cells and functions by phosphorylating various target substrates and thus subsequently affects cell survival, cell proliferation, transcriptional activation, and protein translation. Major studies in cancer cell signaling demonstrated that PIM-2 kinase is intimately involved in the survival and proliferation of different cancer cell types, such as B cell lymphoma (5), multiple myeloma (6), and prostate cancer $(7,8)$, suggesting that it could be a potential therapeutic target for cancer therapy. Despite a growing interest in targeting PIM-2 kinase, there is not yet a specific PIM-2 inhibitor in the clinic, possibly owing to specificity issues relating to the similar structure of PIM isoforms (9). pan-PIM inhibitors were evaluated in several clinical trials, but have been shown to have limited efficacy, possibly pertaining to their ability to inhibit all 3 isoforms (10-12).

The activation of PIM-2 kinase can occur via signal transduction JAK/STAT pathways (Janus kinase and signal transducer and activator of transcription) and subsequently regulate the activity of its downstream effectors (13). The prosurvival protein BAD (Bcl-2associated death promoter) has been identified as one of the PIM-2 target substrates. Phosphorylation of BAD by PIM-2 kinase on

Conflict of interest: The authors have declared that no conflict of interest exists Submitted: May 31, 2017; Accepted: March 29, 2018.

Reference information: J Clin Invest. 2018;128(7):2787-2801.

https://doi.org/10.1172/JCI95407.
Ser-112 reverses BAD-induced cell death and inhibits apoptosis in hematopoietic cell lines (14). PIM-2 kinase has also been implicated in regulating glycolysis by phosphorylating PKM2 (pyruvate kinase M2), which increases the rate of glycolysis and proliferation in cancer cells (15). PIM-2 overexpression in colorectal cancer cells led to increased glycolysis and energy production (16). In addition, inhibition of PIM-2 kinase significantly reduced the growth of multiple myeloma cells $(6,17)$. This mechanism is thought to occur in part by PIM-2 phosphorylating TSC2, a negative regulator of mTORC1. Furthermore, a recent study has demonstrated that treatment with a novel PIM-2 inhibitor (JP11646) suppressed multiple myeloma cell proliferation and reduced tumor growth in xenogeneic myeloma mouse models (18).

Alternatively, PIM-2 kinase is able to promote cell cycle arrest and apoptosis under certain circumstances. A previous study revealed that overexpressing PIM-2 in HeLa cells increased apoptosis in a p73-dependent manner, as coexpression of PIM-2 with a dominant-negative form of p73 abrogated this phenotype (19). Moreover, PIM-2 kinase is able to inhibit cell proliferation through phosphorylation of the cell cycle inhibitor p21Cip1/WAF1 (p21) and enhances p21 stability in HCT116 cells (20).

Very little is known about how PIM-2 activity influences primary T cells, as previous reports of its role are unclear. PIM-1 and PIM-2 kinases are required for rapamycin resistance upon $\mathrm{T}$ cell activation; therefore, the lack of these kinases in vivo has been shown to promote rapamycin sensitivity (21). In addition, the previous report demonstrated that the phosphorylation of Foxp3 by PIM-2 kinase decreased suppressive functions of regulatory T cells (Tregs) (22). In contrast, PIM-2 kinase was previously shown to act as a negative regulator of suppressor of cytokine signaling 1 (SOCS-1) in T cells, and the phosphorylation protects SOCS-1 from degradation (23). 

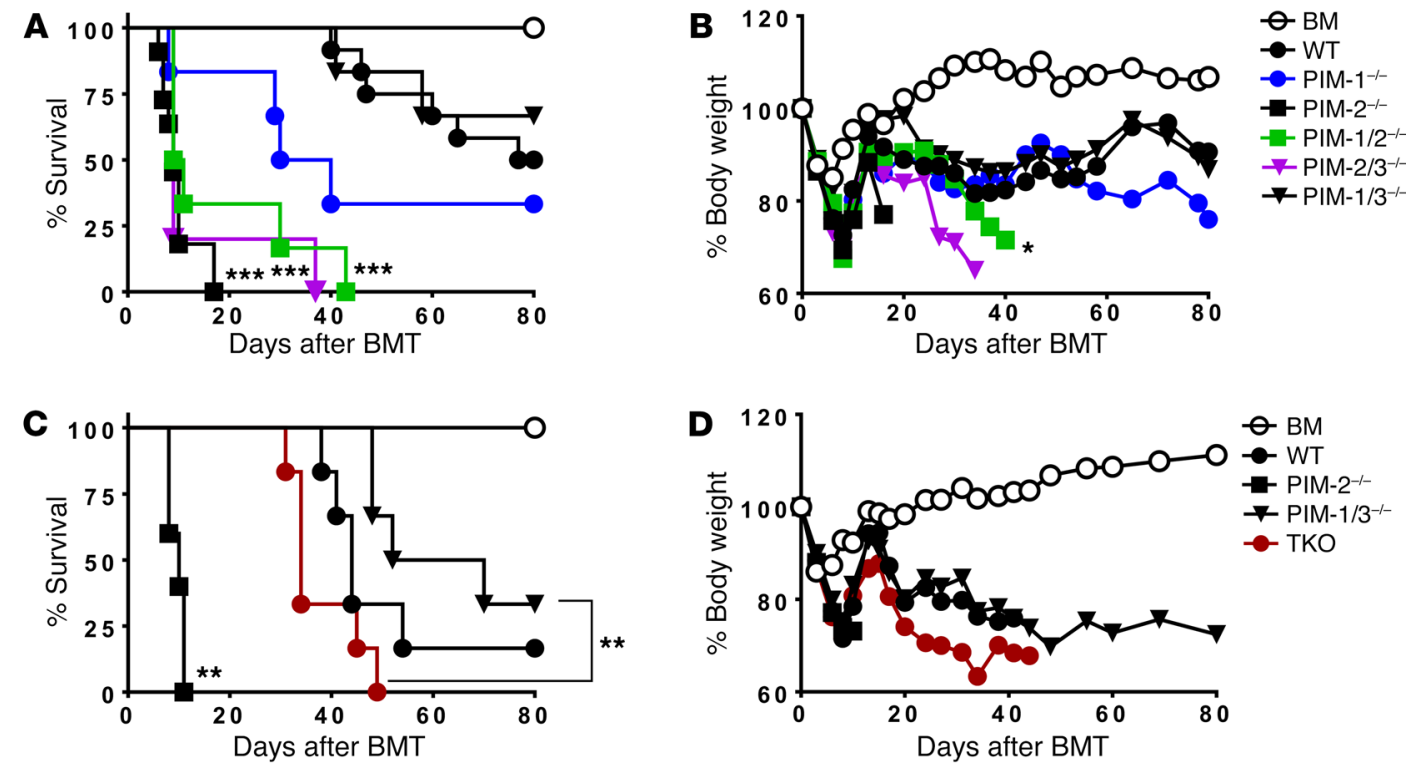

Figure 1. Distinct roles of PIM kinases in T cell alloresponses after BMT. WT B6 mice were lethally irradiated at $950-1,000$ cGy. These recipients then underwent transplantation with WT TCD-BM alone or plus $2 \times 10^{6}$ total T cells isolated from WT, PIM-1 $1^{-1-}, \mathrm{PIM}-2^{-1-}, \mathrm{PIM}-1 / 2^{-1-}, \mathrm{PIM}-1 / 3^{-/-}, \mathrm{PIM}-2 / 3^{-1-}$ double-KO, or PIM-1/2/3 triple-KO (TKO) on an FVB background. Recipient mice were monitored for survival (A and $\mathbf{C}$ ) and body weight changes (B and $\mathbf{D})$ over time. The data shown in $\mathbf{A}$ and $\mathbf{B}$ were pooled from 2 replicate experiments ( $n=10-12$ per group), while the data in $\mathbf{C}$ and $\mathbf{D}$ were obtained from 1 experiment ( $n=5-6$ per group). Significance was determined by log-rank test. ${ }^{*} P<0.05,{ }^{* *} P<0.01,{ }^{* * *} P<0.001$.

In the current work, we attempted to understand how PIM kinases regulate $\mathrm{T}$ cell responses using preclinical models of allogeneic bone marrow transplantation (allo-BMT) and syngeneic models of solid tumors. We demonstrated that PIM-2-deficient T cells undergo robust activation upon $\mathrm{T}$ cell receptor (TCR) stimulation. After being transferred into preconditioned allogeneic recipients, PIM- $2^{-/-}$T cells exhibit enhanced Th1 differentiation, expansion, and migration to target organs resulting in accelerated acute graft-versus-host disease (GVHD) severity. Mechanistic studies revealed that PIM-2-deficient T cells downregulated SOCS-1 and p73 and upregulated IL-9R after allogeneic stimulation, which promoted $\mathrm{T}$ cell survival, proliferation, and proinflammatory cytokine production. Mice deficient in PIM-2 rejected syngeneic tumors primarily through CD8-mediated antitumor responses. Furthermore, adoptive transfer of polyclonal or antigen-specific CD8 ${ }^{+} \mathrm{T}$ cells in which PIM-2 kinase was either deficient or silenced demonstrated superior antitumor activity. In addition, the enhanced IL-9R expression and signaling in PIM-2-- $\mathrm{T}$ cells may contribute to augment their antitumor activity. Taken together, this is, to our knowledge, the first study to show the unique role of the PIM-2 kinase in $\mathrm{T}$ cell responses to alloantigen and tumor cells.

\section{Results}

Distinct roles of PIM kinases in $T$ cell alloresponse after BMT. PIM kinases promote tumor growth by accelerating cell cycle progression and reducing cell apoptosis $(24,25)$. Therefore, PIM inhibitors have been proposed as a means for controlling tumor growth in both solid and hematologic malignancy $(10,26)$. While little is known about the mechanism by which the PIM kinases can regulate immune responses in primary $\mathrm{T}$ cells, Fox et al. demonstrated that the PIM kinases can control the survival and activation of primary $\mathrm{T}$ cells that are resistant to rapamycin (21), an inhibitor of
mTOR and a well-known immunosuppressive drug used in solidorgan as well as allo-BMT. However, administration of rapamycin alone is minimally effective to control GVHD (27). Therefore, we hypothesized that PIM kinase activity may promote $\mathrm{T}$ cell alloresponse in parallel with mTOR and that inhibition of PIM would synergize with rapamycin to control GVHD development. To address this question, we used genetically mutant mice on an FVB background that are deficient for either single, double, or triple kinase of the PIM family. T cell development in thymus was not affected by any PIM kinases as shown previously (28). We also verified that there was no significant difference in $\mathrm{T}$ cell composition such as $\mathrm{CD}^{+}, \mathrm{CD} 8^{+}$, and regulatory $\mathrm{T}$ cells in peripheral lymphoid organs across various strains (Supplemental Figure 1, A-C, and data not shown; supplemental material available online with this article; https://doi.org/10.1172/JCI95407DS1).

To test the role of PIM kinases in the ability of T cells to induce GVHD, we transferred WT, PIM-1 single-KO (PIM-1-1-), PIM-2 single-KO (PIM-2--), PIM-1/2 double-KO (PIM-1/2-/), PIM-1/3 double-KO (PIM- $1 / 3^{-/}$), PIM- $2 / 3$ double-KO (PIM-2/3 ${ }^{-/}$), and PIM- $1 / 2 / 3$ triple-KO (PIM- $1 / 2 / 3^{-/}$) $\mathrm{T}$ cells together with WT bone marrow into lethally irradiated $\mathrm{C} 57 \mathrm{BL} / 6\left(\mathrm{~B} 6, \mathrm{H}-2^{\mathrm{b}}\right)$ mice. As reflected by mortality and loss of body weight after BMT, $\mathrm{PIM}^{-2^{--} \mathrm{T}}$ cells induced more severe GVHD compared with WT $\mathrm{T}$ cells. Furthermore, PIM- $1 / 2^{-/-} \mathrm{T}$ cells were more pathogenic than PIM-1 ${ }^{-/-}$(Figure $1, \mathrm{~A}$ and B), and PIM-1/2/3-/- T cells were more pathogenic than PIM-1/3-/, in the induction of GVHD (Figure 1, $\mathrm{C}$ and $\mathrm{D}$ ). These results suggest a dominant role of PIM-2 kinase in regulating $\mathrm{T}$ cell pathogenicity in GVHD induction and reveal a previously undefined role of PIM-2 kinase, which potently inhibits T cell alloresponses and GVHD induction distinct from PIM- 1 and PIM-3 isoforms. Hereafter, we focused our study on PIM- $-2^{-/-}$with WT and PIM- $1 / 3^{-/-} \mathrm{T}$ cells as controls. 

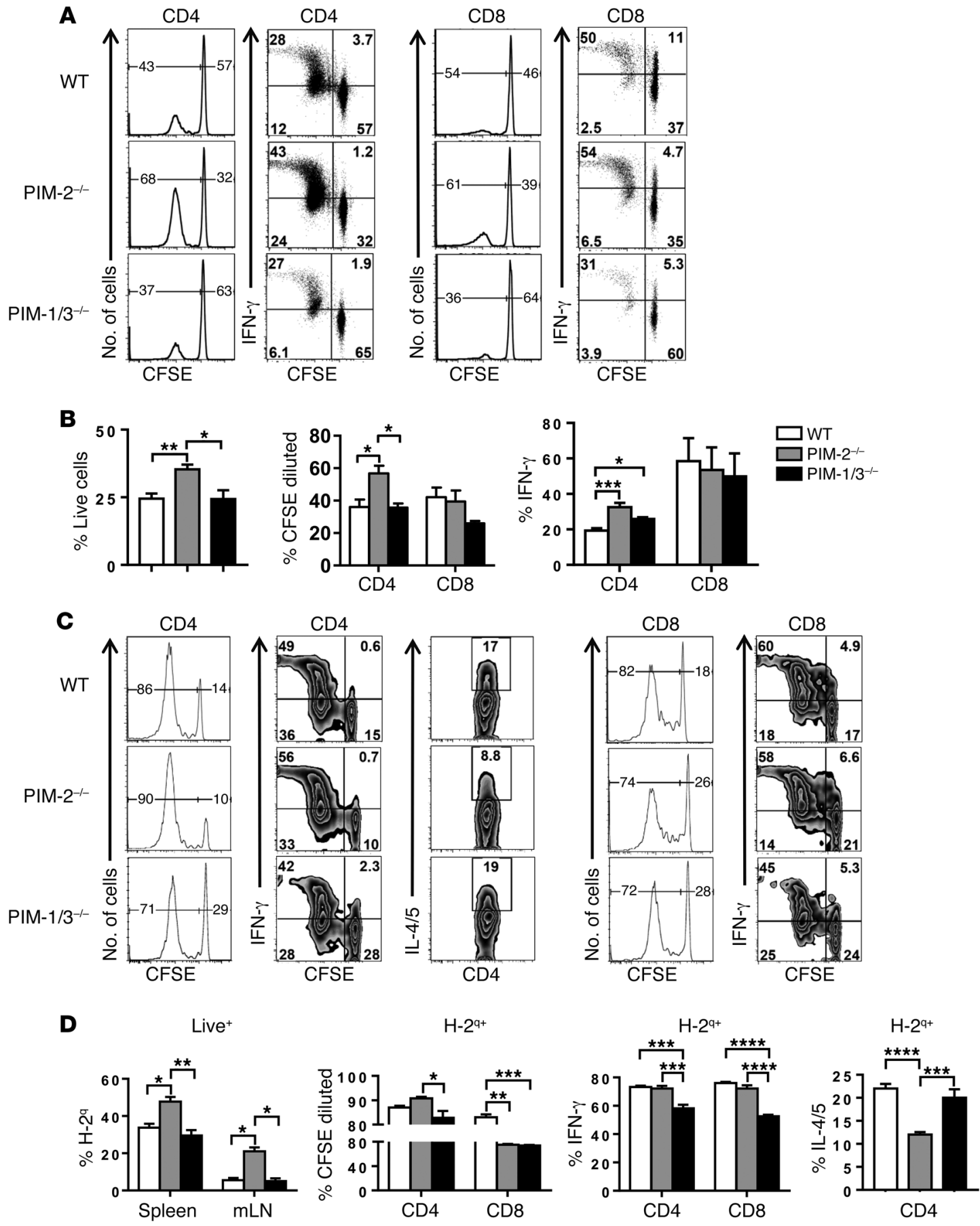

Figure 2. PIM-2 expression inhibits T cell proliferation and Th1 differentiation under allogeneic stimulation in vitro and in vivo. (A and B) In vitro mix lymphocyte reaction. Purified T cells of WT, PIM-2 $2^{-1-}$, and PIM-1/3/-- mice on an FVB background $\left(\mathrm{H}-2^{9}\right)$ were labeled with CFSE and cocultured with T celldepleted splenocytes as antigen-presenting cells from $\mathrm{B} 6$ mice $\left(\mathrm{H} 2^{\mathrm{b}}\right)$ for 5 days. Cells were restimulated with PMA and ionomycin for cytokine secretion. Percentages of CFSE-diluted and IFN- $\gamma$-producing cells on gated live donor CD4+ or CD8 ${ }^{+}$T cells $(n=6)$. (C) Purified T cells from WT, PIM-2 ${ }^{-1-}$, and PIM-1/3 $3^{-1-}$ mice were labeled with CFSE and transferred into lethally irradiated BALB/c $\left(\mathrm{H}-2^{\mathrm{d}}\right)$ mice at $2 \times 10^{6}$ cells per mouse. Four days after cell transfer, recipient spleens and $\mathrm{mLNs}$ were harvested and analyzed by flow cytometry. Representative figures and percentages are shown on gated live cells followed by $\mathrm{H}-2^{\mathrm{q}+}$ cells. (D) Percentages of donor T cells are shown in recipient spleen and mLNs. Average percentages of CFSE-diluted, IFN- $\gamma^{+}$, IL-4/5+ cells are shown on gated live donor $\mathrm{CD}^{+}$or $\mathrm{CD} 8^{+} \mathrm{T}$ cells in recipient spleen ( $n=4-5$ mice per group). Data are representative of at least 2 independent experiments and are shown as mean \pm SEM by 1-way ANOVA and Tukey's HSD post hoc analysis (B and $\mathbf{D})$. ${ }^{*} P<0.05,{ }^{* *} P<0.01,{ }^{* *} P<0.001,{ }^{* * *} P<0.0001$.

PIM-2 expression inhibits T cell proliferation and Th1 differentiation under allogeneic stimulation both in vitro and in vivo. To further evaluate the effect of the PIM-2 kinase in T cell homeostasis, we compared $\mathrm{T}$ cell composition and phenotype in WT, $\mathrm{PIM}-2^{-/}$, and $\mathrm{PIM}-1 / 3^{-/-}$
$\left(\mathrm{H}-2^{\mathrm{q}}\right)$ mice. Because of its relevance to GVHD induction $(29,30)$, we also measured the memory subsets of the T cell compartment. Percentages of naive or memory $\mathrm{T}$ cells were comparable regardless of PIM expression (Supplemental Figure 1D). The frequencies of B 
A

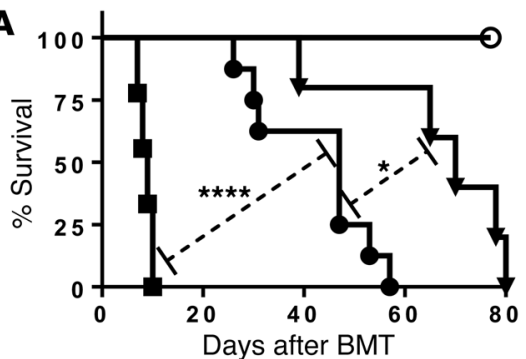

B

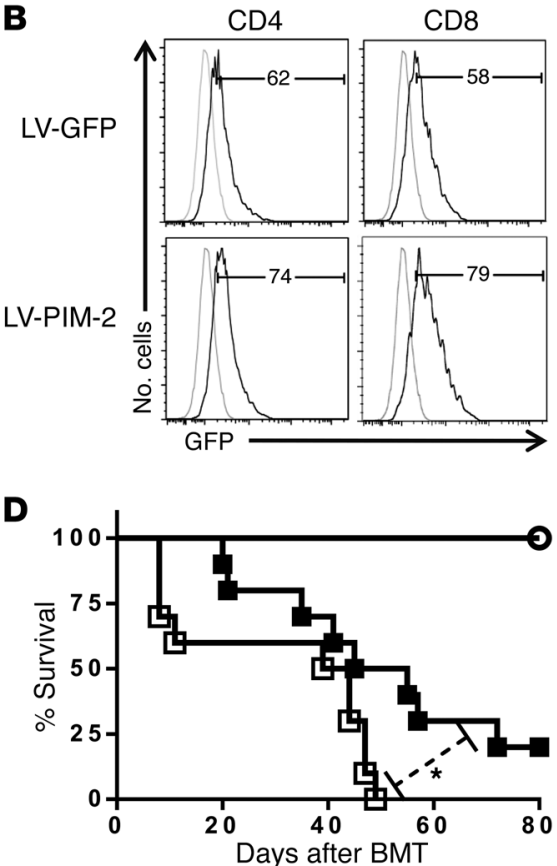

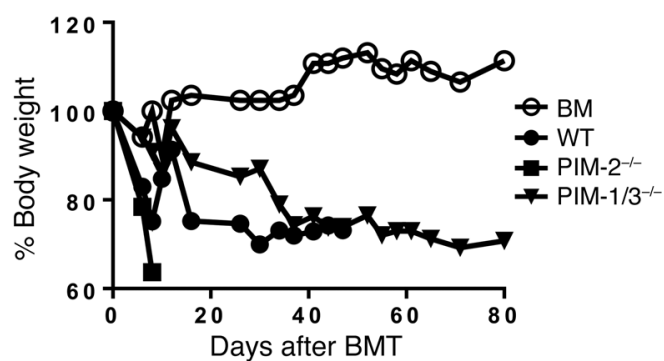

C
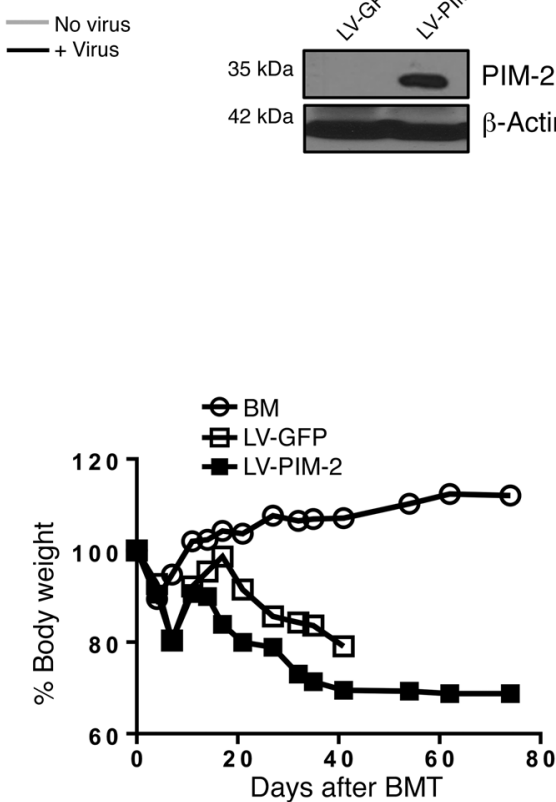

Figure 3. PIM-2 kinase suppresses T cell activity to induce acute GVHD. (A) Lethally irradiated BALB/C (700 cGy) mice underwent transplantation with $5 \times 10^{6}$ TCD-BM per mouse plus $2 \times 10^{5} \mathrm{~T}$ cells per mouse isolated from WT, PIM-2-1-, and PIM-1/3/- FVB donors. Survival and body weight loss were monitored ( $n=5-10$ mice per group). (B) Histogram plots show percentages of transduction efficiency as depicted by percent GFP' cells. (C) PIM-2 $2^{-1-}$ T cells transduced with LV-PIM-2-GFP were harvested 48 hours after transduction and analyzed for Western blot. LV-GFP-transduced cells were used as control. (D) Purified T cells from $\mathrm{PIM}-2^{-/-}$mice were stimulated with antiCD3 $(5 \mu \mathrm{g} / \mathrm{ml})$ and anti-CD28 $(5 \mu \mathrm{g} / \mathrm{ml})$. After 48 hours of activation, $T$ cells were transduced with lentivirus encoding GFP or PIM-2-GFP. Twenty-four hours after transduction, $3 \times 10^{6}$ activated T cells per mouse were transferred into lethally irradiated BALB/C mice with $5 \times 10^{6} \mathrm{TCD}-\mathrm{BM}$ per mouse. Survival and body weight loss were monitored ( $n=10$ mice per group). Data are representative of 2 independent experiments, and significance was determined by log-rank test. ${ }^{*} P<0.05,{ }^{* * *} P<0.0001$ cells $\left(\mathrm{B} 22 \mathrm{O}^{+}\right)$, dendritic cells (CD11 $\left.\mathrm{c}^{+}\right)$, and myeloid-derived suppressor cells $\left(\mathrm{CD} 11 \mathrm{~b}^{+} \mathrm{Gr}-1^{+}\right)$were similar among different strains (data not shown). However, the size of the NK cell population (NK1.1 $1^{+}$) was lower in PIM mutant mice (Supplemental Figure 1E).

We then measured $\mathrm{T}$ cell activation and proliferation upon alloantigen stimulation in vitro. As reflected by CFSE dilution and IFN- $\gamma$ production, PIM-2-- $\mathrm{CD}^{+} \mathrm{T}$ cells showed a significant increase in T cell proliferation compared with $\mathrm{WT}$ and PIM-1/3 ${ }^{-/-}$ $\mathrm{CD} 4^{+} \mathrm{T}$ cells, whereas PIM-2-- $\mathrm{CD}^{+} \mathrm{T}$ cells proliferated similarly to WT but more than PIM-1/3-1- CD $8^{+} \mathrm{T}$ cells (Figure 2, $\mathrm{A}$ and $\mathrm{B}$ ). Moreover, IFN- $\gamma$ production of WT CD $4^{+} \mathrm{T}$ cells was substantially lower than that of PIM-2-- $\mathrm{CD}^{+} \mathrm{T}$ cells; however, no difference was observed in IFN- $\gamma$ production of $\mathrm{CD}^{+} \mathrm{T}$ cells between these 3 groups. These data suggest that PIM-2 kinase suppresses CD4 $4^{+} \mathrm{T}$ cell proliferation and differentiation to Th1 cells in vitro.

To further evaluate the role of PIM-2 kinase in T cells in vivo, $\mathrm{PIM}-2^{--} \mathrm{T}$ cells isolated from FVB donors were transferred into irradiated allogeneic BALB/c recipients $\left(\mathrm{H}-2^{\mathrm{d}}\right)$. Four days after allogeneic stimulation, donor T cells $\left(\mathrm{H}-2^{\mathrm{q}}\right)$ were harvested from spleen and mesenteric lymph node (mLN). Compared with controls, an increased frequency of PIM- $2^{--}$donor $\mathrm{T}$ cells was observed in the spleen and mLN, suggesting that PIM-2- ${ }^{--} \mathrm{T}$ cells had higher proliferation ability in vivo as well as increased migration to both the gut and draining lymph nodes. As reflected by
CFSE dilution, $\mathrm{PIM}-2^{-{ }^{-}} \mathrm{CD} 4^{+} \mathrm{T}$ cells proliferated faster in vivo compared with PIM- $1 / 3^{-1-} \mathrm{T}$ cells although there was no difference from WT T cells (Figure 2, C and D). In this short-term response, PIM-2- ${ }^{--} \mathrm{CD} 4^{+} \mathrm{T}$ cells produced similar levels of IFN $-\gamma$ but considerably lower levels of IL-4/5 compared with WT and PIM-1/3-1$\mathrm{CD}^{+} \mathrm{T}$ cells. On the other hand, PIM- $1 / 3^{-/-} \mathrm{T}$ cells exhibited a marked decrease of IFN- $\gamma$ production in both $\mathrm{CD}^{+}$and $\mathrm{CD}^{+} \mathrm{T}$ cells, suggesting that PIM-1 and PIM-3 isoforms are required for Th1 and Tc1 polarization in vivo.

PIM-2 kinase suppresses T cell ability to induce acute GVHD. To assess the role of PIM-2 kinase in GVHD induction, we carried out allo-BMT from FVB into BALB/c. Consistent with the results in B6 recipients (Figure 1), PIM-2-- $\mathrm{T}$ cells induced lethal acute GVHD in BALB/c recipients within 10 days after BMT. The recipient mice had a significantly higher body weight loss with bloody diarrhea (Figure 3A). To better quantify the pathogenicity of PIM-2cells, we titrated the number of $\mathrm{T}$ cells needed for the induction of acute GVHD and found that as few as 50,000 PIM-2-- $\mathrm{T}$ cells could still induce lethal acute GVHD (Supplemental Figure 2A). Given that such a low number of WT T cells are incapable of inducing acute GVHD (Supplemental Figure 2B), these data highlight the increased activity of PIM-2-- T cells in acute GVHD induction. To exclude the possibility that the pathogenicity of PIM- $2^{-/-} \mathrm{T}$ cells was independent of allorecognition, we transferred these cells to 
A
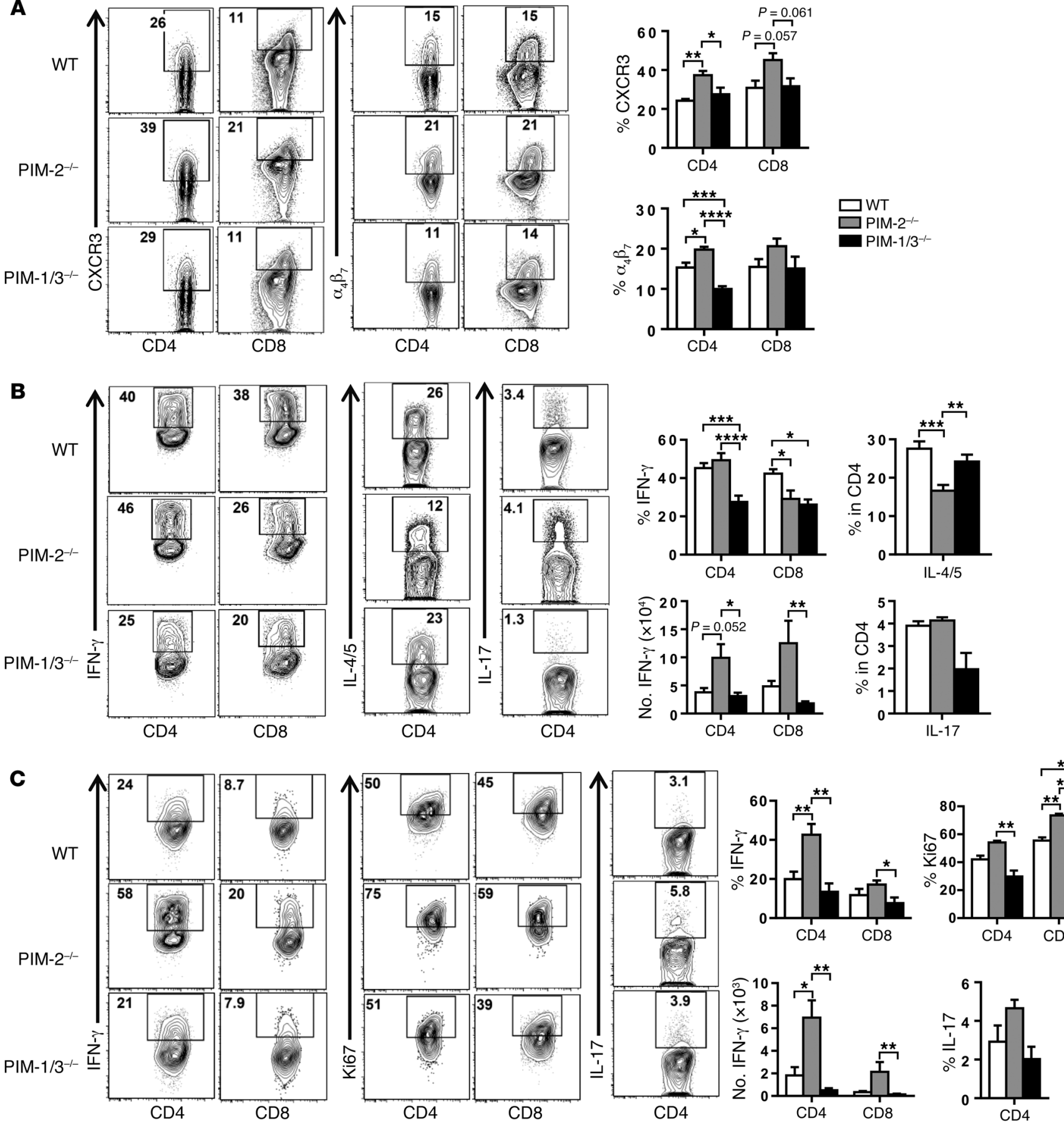

Figure 4. PIM-2 kinase suppresses T cell differentiation into Th1 cells, T cell migration, and GVHD pathogenicity. (A-C) Seven days after GVHD induction, spleen, liver, and gut were collected from the recipient mice, and the resulting cell suspension was analyzed by flow cytometry. (A) Representative dot plots show percentages of CXCR3 and $\alpha_{4} \beta_{7}$ expression on gated donor CD4 $4^{+}$or CD8 ${ }^{+}$T cells in recipients' spleens. (B) The percentages of IFN- $\gamma-$, IL-4/5-, or IL-17secreting cells are shown on gated donor $\mathrm{CD}^{+}$and $\mathrm{CD}^{+} \mathrm{T}$ cells in recipients' livers. The absolute number of IFN- $\gamma$ among donor T cells is depicted. (C) The frequency of IFN- $\gamma$, IL-4/5, IL-17, and Ki67 among isolated donor T cells from recipients' intestines. Data are representative of 2 independent experiments ( $n=$ 7-10 mice per group) and are shown as mean \pm SEM by 1-way ANOVA and Tukey's HSD post hoc analysis. ${ }^{*} P<0.05,{ }^{* *} P<0.01,{ }^{* * *} P<0.001,{ }^{* * * *} P<0.0001$.

syngeneic FVB recipients. Similarly to WT or PIM-1/3-/- T cells, as many as $2 \times 10^{6} \mathrm{PIM}-2^{-/-} \mathrm{T}$ cells failed to induce any signs of GVHD (Supplemental Figure 2C).

To address whether the absence of PIM-2 was truly responsible for the enhanced ability of T cells to cause acute GVHD, we investigated whether restoring PIM-2 expression on $\mathrm{PIM}-2^{-/-} \mathrm{T}$ cells could alleviate GVHD severity. To accomplish this experiment, preactivated $\mathrm{T}$ cells were transduced with a lentivirus expressing PIM-2/GFP or GFP only as a control vector. Similar levels of virus transduction, $60 \%-70 \% \mathrm{GFP}^{+}$cells, were obtained in both cell types (Figure 3B). The expression level of PIM-2 protein was also confirmed by Western blot analysis (Figure 3C). Importantly, 
T cells transduced with PIM-2-expressing vector had a reduced ability to induce GVHD (Figure 3D) compared with PIM-2-- T cells transduced with empty vector. Taken together, these results clearly demonstrated that PIM-2 potently suppressed T cell activation and function in the induction of acute GVHD.

Because several pan-PIM inhibitors have been developed to treat multiple types of cancer, as a result, we have also studied the effect of the pan-PIM inhibitor AZD1208 in GVHD models. AZD1208 was shown to inhibit all 3 PIM isoforms with $\mathrm{IC}_{50}$ of $0.4 \mathrm{nM}, 5 \mathrm{nM}$, and 1.9 nM for PIM-1, PIM-2, and PIM-3, respectively (10).

Using AZD1208 inhibitor, the treatment of recipients that were transplanted with WT T cells could significantly attenuate GVHD, consistent with previous studies in tumors using this inhibitor (Supplemental Figure 3, A and B). Our interpretation is that preferential inhibition of PIM-1 and PIM-3 isoforms by AZD1208 resulted in GVHD alleviation. On the other hand, pan-PIM inhibition did not protect the recipients of PIM-2-/- T cells from GVHD (data not shown), which could be due to the extremely severe GVHD in these recipients. Strikingly, inhibition of PIM-2 isoform in PIM-1/3-deficient T cells with the inhibitor induced higher T cell activity and accelerated GVHD severity, with more than $75 \%$ mouse mortality within 10 days, similar to the results with genetically knocked out T cells (Figure 3A).

PIM-2 kinase suppresses Th1 differentiation, migration, and GVHD pathogenicity. To understand the underlying mechanisms by which PIM-2- ${ }^{--}$T cells induced severe GVHD, we further characterized PIM-2 ${ }^{-/-} \mathrm{T}$ cells in the recipients after BMT. Donor T cells were recovered 7 days after BMT from recipients' spleen, liver, and intestine. We measured the expression of chemokine receptor CXCR3 and integrin receptor $\alpha_{4} \beta_{7}$, as their expression promotes $\mathrm{T}$ cell migration to GVHD target organs $(31,32)$. Indeed, both CXCR3 and $\alpha_{4} \beta_{7}$ expression was upregulated in PIM-2 $2^{-/}$donor $\mathrm{CD}^{+} \mathrm{T}$ cells in recipients' spleens (Figure $4 \mathrm{~A}$ ). Given that the generation of induced Tregs (iTregs) is associated with the severity of GVHD, we also measured iTreg generation in recipients' spleens. However, we found that the percentages of iTregs in recipients were comparable regardless of donor $\mathrm{T}$ cell type (data not shown).

To further elucidate the role of PIM-2 kinase in GVHD pathogenesis, donor $\mathrm{T}$ cells isolated from target organs, the liver and gut, were examined. PIM-2-/- CD4 ${ }^{+} \mathrm{T}$ cells produced levels of IFN- $\gamma$ similar to those produced by WT CD $4^{+} \mathrm{T}$ cells, but lower levels of IL- $4 / 5$ than WT and PIM- $1 / 3^{-/-}$counterparts, in the liver of recipient mice. In contrast, the percentage of IFN- $\gamma$-secreting $\mathrm{CD}^{+} \mathrm{T}$ cells was decreased in the PIM-2/- group. However, the absolute numbers of $\mathrm{CD}^{+}$and $\mathrm{CD}^{+} \mathrm{T}$ cells that produced IFN- $\gamma$ were substantially increased in the liver of $\mathrm{PIM}-2^{-/-}$recipients (Figure 4B and Supplemental Figure 4B), suggesting an increase of $\mathrm{T}$ cell infiltration into the GVHD target organs. Similarly, PIM-2 $2^{-/-} \mathrm{T}$ cells isolated from the intestine of recipients produced substantially higher levels of IFN- $\gamma$ and Ki67 expression, a marker of cell proliferation. Moreover, given the important role of IL-17 in gut GVHD (33), we measured IL-17 production, and no difference was observed between these experimental groups with respect to IL-17 expression in the liver and gut (Figure 4C). A modest increase in absolute cell numbers was also observed in the intestine of $\mathrm{PIM}-2^{-/-}$recipients. The increased absolute $\mathrm{T}$ cell numbers in the recipients' liver and intestine were conversely cor- related with the total cell number in spleen (Supplemental Figure 4B). We reason that PIM-2/- donor T cells were activated strongly in recipient spleens and then rapidly migrated to target organs in conjunction with $\mathrm{T}$ cell expansion locally, which resulted in severe GVHD. In addition, the percentage of dead cells among PIM-2-/- $\mathrm{CD}^{+} \mathrm{T}$ cells was significantly decreased compared with WT (Supplemental Figure 4A), suggesting that PIM-1 and PIM-3 kinases are essential for $\mathrm{CD} 8^{+} \mathrm{T}$ cell survival.

Given the high pathogenicity of PIM-2-/- T cells in GVHD, we further hypothesized that PIM-2-/- T cells would be more efficacious in mediating a graft-versus-leukemia (GVL) response. To test this hypothesis, we used A20, a B cell lymphoma on the BALB/C background. Fifty thousand $\mathrm{T}$ cells per mouse were injected into $\mathrm{BALB} / \mathrm{c}$ recipients to avoid induction of lethal GVHD. The recipients with transplanted BM and A20 lymphoma alone succumbed to tumor relapse by day 20, whereas the mice that received either A20 plus WT or PIM-1/3/- T cells had tumor relapse by day 26 . In contrast, $\mathrm{PIM}-2^{--} \mathrm{T}$ cells were remarkably superior in mediating the GVL response, as reflected by a substantial lower tumor burden measured with bioluminescent imaging in addition to a delay in tumor relapse (Supplemental Figure 5, A and B).

We further evaluated the GVL effect in a clinically relevant model against $\mathrm{GFP}^{+}$MLL-AF9 (34). T cell dose was reduced to 25,000 T cells to avoid severe GVHD and early death in mice with transplanted $\mathrm{PIM}-2^{-/-} \mathrm{T}$ cells. In fact, the $\mathrm{T}$ cell dose was 8-fold lower than a usual dose of T cells $\left(2 \times 10^{5} \mathrm{~T}\right.$ cells $)$ used in this model. We observed a significant delay of tumor growth measured by lower percentages of $\mathrm{GFP}^{+}$cells in peripheral blood on days 21, 28, and 35 after allo-BMT (Figure 5, A and B). Moreover, PIM-2-/- T cells could significantly delay the tumor growth and prolonged recipient survival from MLL-AF9 (Figure 5C). These data indicated that PIM-2 $2^{-1-} \mathrm{T}$ cells were more effective to control MLL-AF9 leukemia than WT T cells.

PIM-2 deficiency promotes host antitumor response. Because PIM-2 deficiency unleashed T cell responses to alloantigen and subsequent induction of super-acute GVHD and superior GVL effect, we further hypothesized that PIM-2 inhibition in T cells could be a potential strategy to augment antitumor activity. To test this hypothesis, we injected syngeneic TS-1 breast tumor cells (35) into WT or PIM-2-- FVB mice and observed that WT mice developed a tumor in the lung and died in 20-40 days. In sharp contrast, the same tumor failed to grow in $\mathrm{PIM}-2^{-/-} \mathrm{FVB}$ mice (Figure $6, \mathrm{~A}-\mathrm{C})$. The same results were observed when tumor cells were implanted subcutaneously (Figure 6D).

To understand the underlying mechanisms, we isolated tumor-infiltrating lymphocytes (TILs) from the lungs of tumorbearing mice and evaluated their phenotype and function. As expected, the TILs in WT tumor-bearing mice had a higher frequency of infiltrating Tregs $\left(\mathrm{TCR} \beta^{+} \mathrm{Foxp} 3^{+}\right)$. Among the TILs, $\mathrm{CD}^{+} \mathrm{T}$ cells also expressed high levels of PD-1, a marker of T cell exhaustion. This exhausted phenotype correlated with downregulation of proinflammatory cytokines such as IFN- $\gamma$, TNF- $\alpha$, and IL-2 $\left(\mathrm{TCR} \beta^{+} \mathrm{CD} 4^{+}\right)$. In contrast to PIM- $2^{-/-} \mathrm{CD} 4^{+} \mathrm{T}$ cells isolated from tumor-bearing mice that expressed lower levels of Foxp 3 and PD-1 and higher levels of IFN- $\gamma$, TNF- $\alpha$, and IL-2, PIM-2-/- CD $8^{+}$ $\mathrm{T}$ cells were observed to produce less IFN- $\gamma$ cytokine. Strikingly, both $\mathrm{CD}^{+}$and $\mathrm{CD} 8^{+} \mathrm{T}$ cells in PIM-2/- mice expressed signifi- 

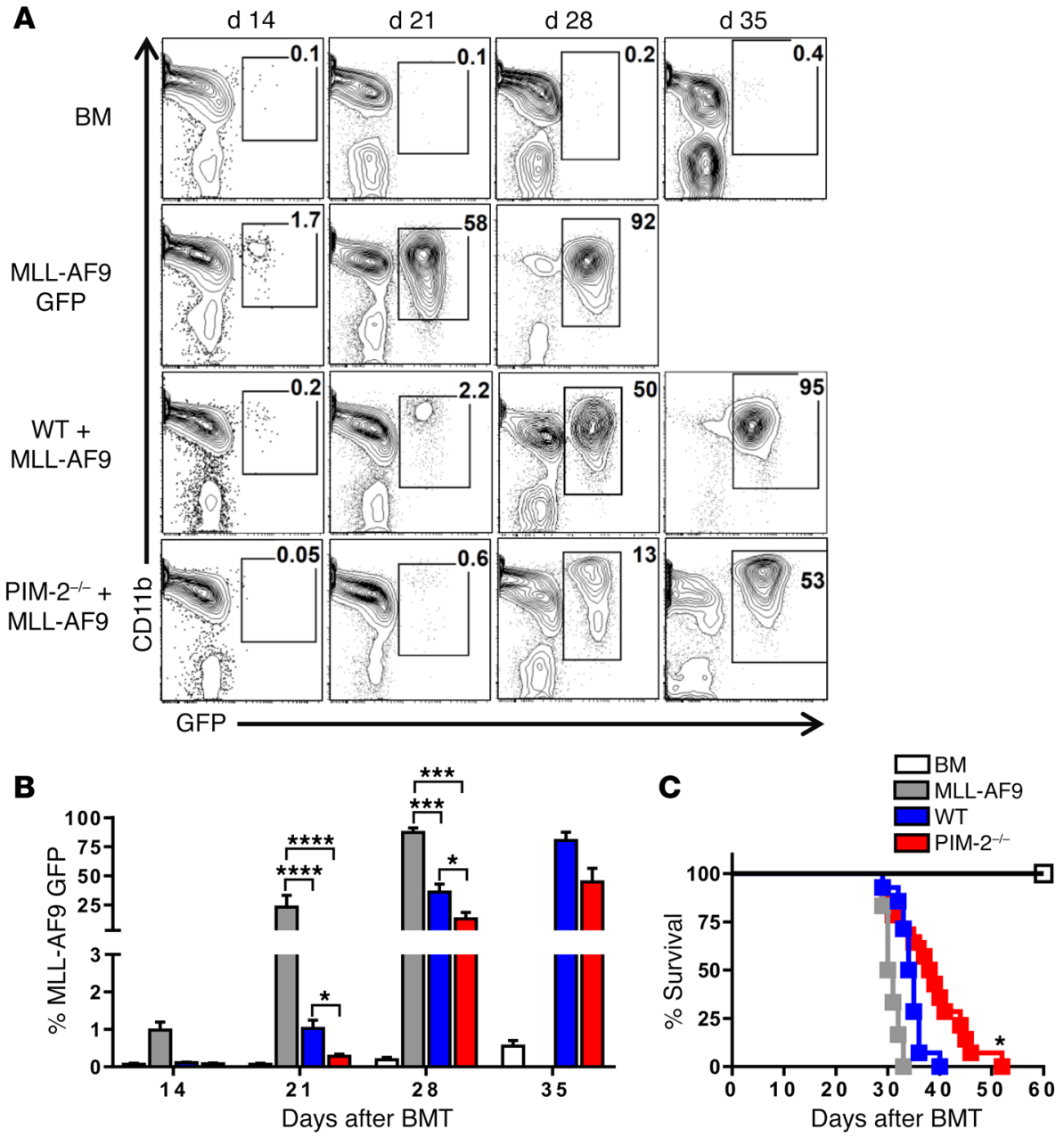

Figure 5. PIM-2-/- T cells mediate GVL effect in MLL-AF9 model. Lethally irradiated (700 cGy) $\mathrm{BALB} / \mathrm{c}$ mice underwent transplantation with $5 \times 10^{6} \mathrm{TCD}$-BM per mouse or BM plus $2.5 \times 10^{4} \mathrm{~T}$ cells per mouse isolated from WT or PIM-2-1- FVB donors and $2 \times 10^{4}$ MLL-AF9-GFP. (A) Percentages of MLL-AF9 cells that were CD11b+CFP+ were analyzed in peripheral blood, and representative dot plots on days 14, 21, 28 and 35 are shown ( $n=14$ mice per group). (B) Bar graph shows quantified GFP percentages of MLL-AF9 in blood at the indicated time points. (C) Survival of recipient mice was monitored ( $n=14$ mice per group). Data are pooled from 2 independent experiments and shown as mean \pm SEM by 1-way ANOVA and Tukey's HSD post hoc analysis (B) and log-rank test (C). ${ }^{*} P<0.05$, ${ }^{* *} P<0.001$, **** $P<0.0001$.

cantly higher levels of FasL than WT T cells, which could greatly contribute to tumor killing in vivo (Figure 6, E and F) (36).

PIM-2 kinase suppresses antitumor immunity primarily mediated by $\mathrm{CD}^{+} \mathrm{T}$ cells. Since PIM-2-/- mice completely rejected TS-1 tumor cells (Figure 7, A-C), one possibility may be that PIM-2-1mice were not genetically identical to tumor cells and thus tumor could be rejected by disparate antigens. To exclude this possibility, we implanted TS- 1 tumor into PIM-2 ${ }^{+/-}$mice and observed that the tumor grew at the same rate as in WT controls (Figure 7B). Together with the observation that syngeneic BMT of PIM-2-/- $\mathrm{T}$ cells did not induce any signs of GVHD (Supplemental Figure 2C), this led us to the interpretation that PIM-2/- mice were of pure FVB background and syngeneic to the TS- 1 tumor. Given the predominant role of $\mathrm{CD}^{+} \mathrm{T}$ cells in antitumor immunity, we next addressed the contribution of $\mathrm{CD}^{+} \mathrm{T}$ cells in tumor rejection in $\mathrm{PIM}-2^{-/-}$mice. Indeed, depletion of $\mathrm{CD}^{+} \mathrm{T}$ cells in $\mathrm{PIM}-2^{-/-}$mice resulted in $100 \%$ tumor relapse and death within 70 days (Figure 7, A and B). These data clearly demonstrated that $\mathrm{CD} 8^{+} \mathrm{T}$ cells played a crucial role in antitumor immunity in $\mathrm{PIM}-2^{-/-}$mice.

We next focused on the ability of PIM-2-/- $\mathrm{CD} 8^{+} \mathrm{T}$ cells to mediate the antitumor response. To examine the activity of these cells, WT tumor-bearing mice were sublethally irradiated, then transferred with purified WT or PIM- $2^{-/-} \mathrm{CD}^{+} \mathrm{T}$ cells (Figure 7C). As expected, tumor-bearing mice that did not receive any $\mathrm{T}$ cells succumbed to tumor relapse rapidly. Consistent with a very low frequency of tumor-specific T cells in syngeneic unprimed $\mathrm{T}$ cells, the mice infused with WT $\mathrm{CD}^{+} \mathrm{T}$ cells had tumor relapse similar to that of those without $\mathrm{T}$ cell transfer. Strikingly, transfer of 2 million PIM-2/- $\mathrm{CD}^{+} \mathrm{T}$ cells could significantly delay tumor relapse (Figure 7D).

To further examine whether this antitumor response could be achieved by silencing of PIM- 2 on T cells, we used lentivirus expressing shRNA to silence PIM- 2 in $\mathrm{CD}^{+} \mathrm{T}$ cells isolated from Pmel-1 TCR-transgenic mice, whose $\mathrm{CD}^{+} \mathrm{T}$ cells express TCR specific to melanoma antigen gp-100. These cells were adoptively transferred to melanoma tumor-bearing mice. Upon PIM-2 silencing, $\mathrm{T}$ cells produced higher levels of TNF- $\alpha$ but a comparable level of IFN- $\gamma$ cytokines (Figure 7E). While the adoptive transfer of Pmel-1 T cells transduced with control vector reduced tumor burden and prolonged mouse survival to some extent, Pmel-1 T cells with silenced PIM-2 were superior to these controls (Figure 7, F and G). These results suggested that PIM-2-silenced $C D 8^{+} \mathrm{T}$ cells are more potent in mediating antitumor immunity.

PIM-2 kinase regulates $T$ cell alloresponses through IL-9/ $I L-9 R$ signaling. To assess the molecular mechanisms pertaining to how PIM-2 negatively regulates T cell responses, we initially performed transcriptional profiling of purified $\mathrm{T}$ cells from WT and $\mathrm{PIM}^{-2^{-/}}$mice after anti-CD3 and anti-CD28 activation. RNA sequencing analysis revealed several potential downstream transcriptional regulators (Figure 8, A and B). PIM-2-/- 
B

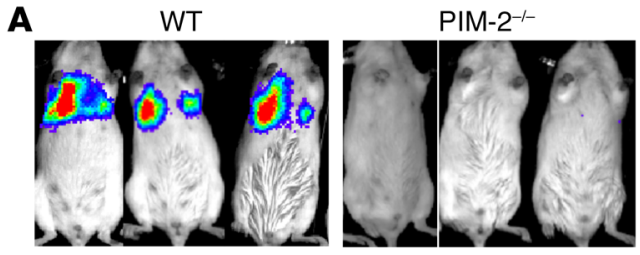

WT

$\mathrm{PIM}-2^{-1-}$
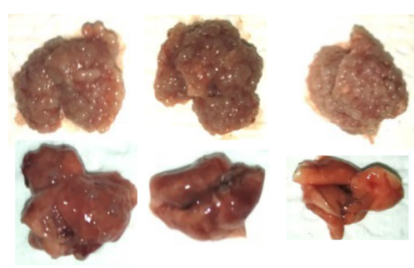

C
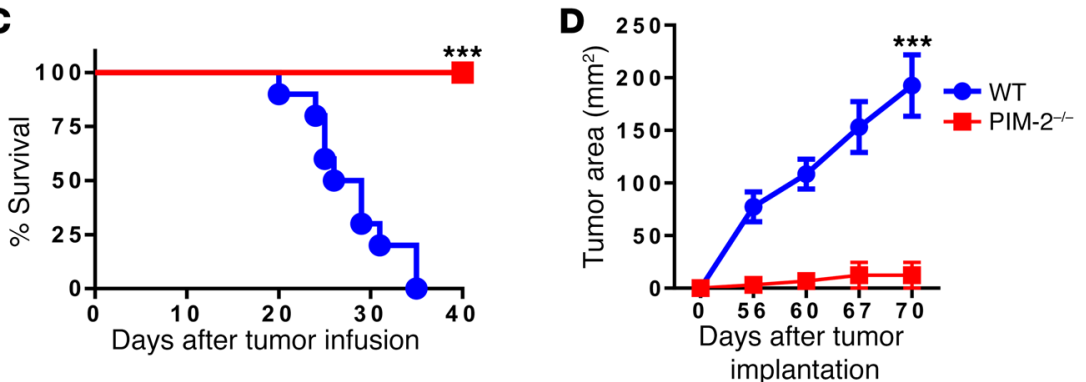

E
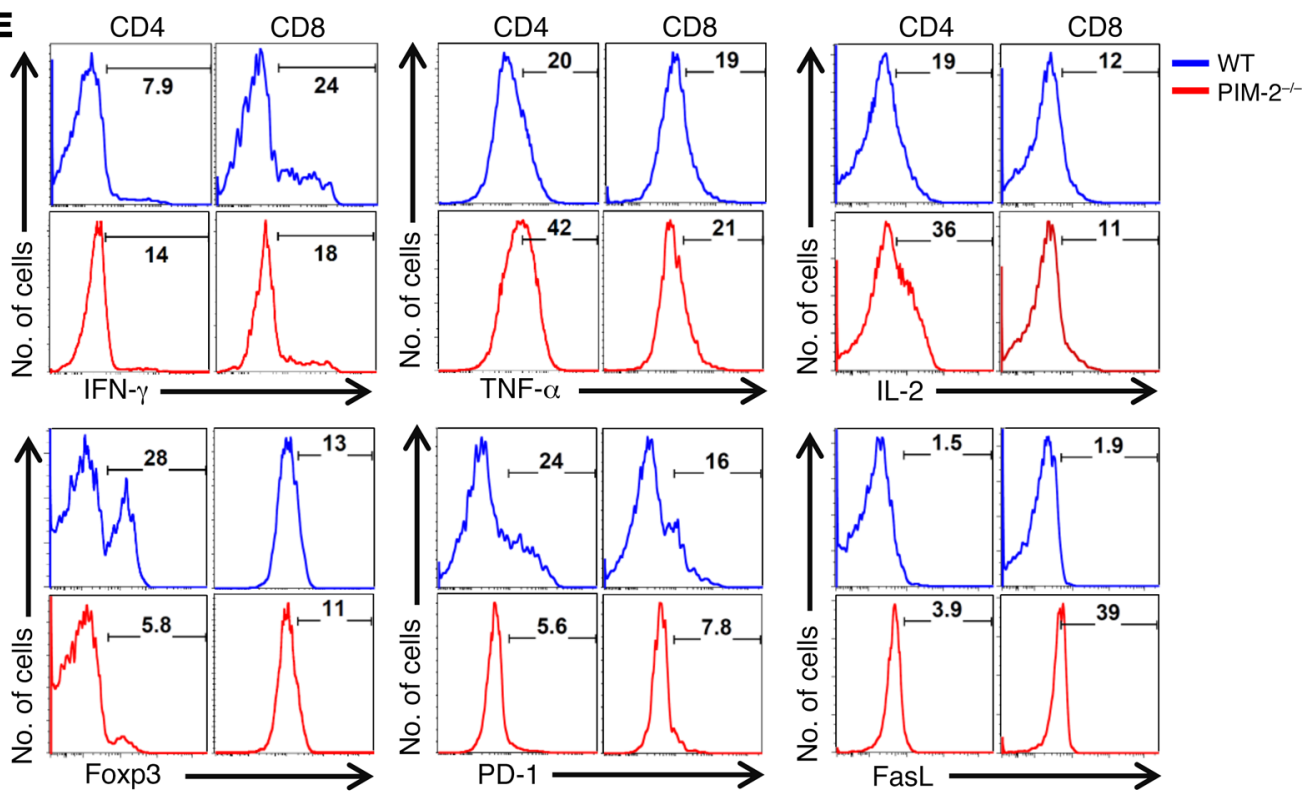

F
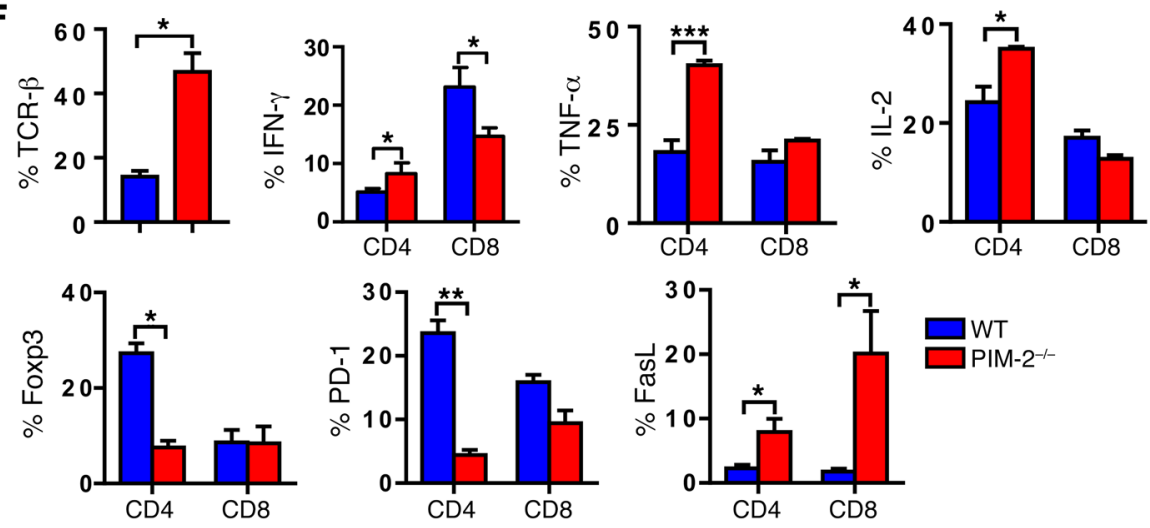

Figure 6. PIM-2 deficiency promotes host antitumor response. WT and PIM-2-/- mice were infused with TS-1 tumor i.v. or s.c. (A) Bioluminescent imaging shows tumor growth in lungs of WT and PIM-

$2^{-/-}$mice. (B) Photographs of lungs from day 27 after tumor infusion. (C) Percentages of mouse survival after tumor infusion i.v. (D) Tumor growth was measured at indicated time points after s.c. implantation ( $n=5$ mice per group). (E) Representative histograms show percentages of IFN- $\gamma$, TNF- $\alpha$, IL-2, Foxp3, PD-1, and FasL expression of $\mathrm{CD} 4^{+}$or $\mathrm{CD} 8^{+} \mathrm{T}$ cells (gated on TCR $\beta^{+}$) in tumor-infiltrating lymphocytes on day 27. (F) Bar graphs show mean percentages of each molecule. Data were pooled from 2 independent experiments ( $n=7$ mice per group). Significance was determined by log-rank test (C) and 2-way ANOVA (D). Data represent mean \pm SEM by 2-tailed Student's $t$ test $(\mathbf{F}) .{ }^{*} P<0.05$, ${ }^{* *} P<0.01,{ }^{* *} P<0.001$.
T cells upregulated JAK1, STAT1, IRF7, IL-2,IFN- $\gamma$, and TNF- $\alpha$ family members, which are important for $\mathrm{T}$ cell survival and signaling. In addition, expression of several memory-associated genes such as TCF7, BCL-2, CCR7, CSF2RA, and EOMES was also increased in PIM-2 $2^{-/} \mathrm{T}$ cells. Higher levels of molecules involved in antitumor responses such as FASL, TBX21, IFN- $\gamma$,
TNF- $\alpha, H D A C 9$, and $I L-9 R$ were also observed (Figure 8A). On the other hand, negative regulators involved in $\mathrm{T}$ cell functions were found to be reduced in PIM-2-/- T cells, including SOCS-1, SOCS-3, CTLA-2A, IL-1O, IL-4, IL-5, SMAD3, and TGF $\beta R 2$ (Figure $8 \mathrm{~B})$. Consistent with previous reports (23), mRNA expression and Western blot analysis showed a decreased level of SOCS-1 in 
A

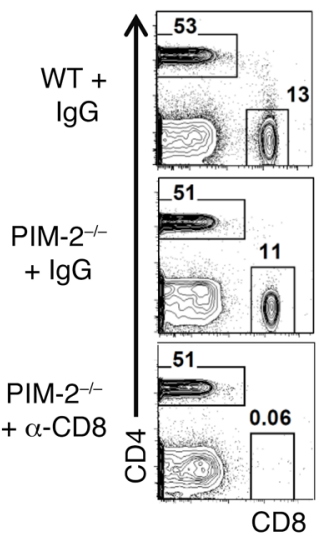

B

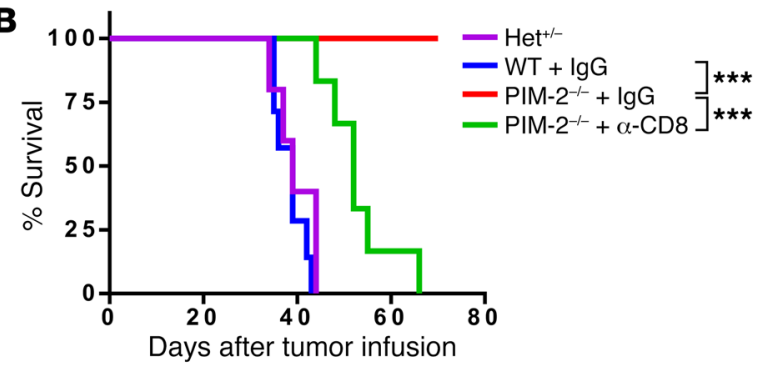

C
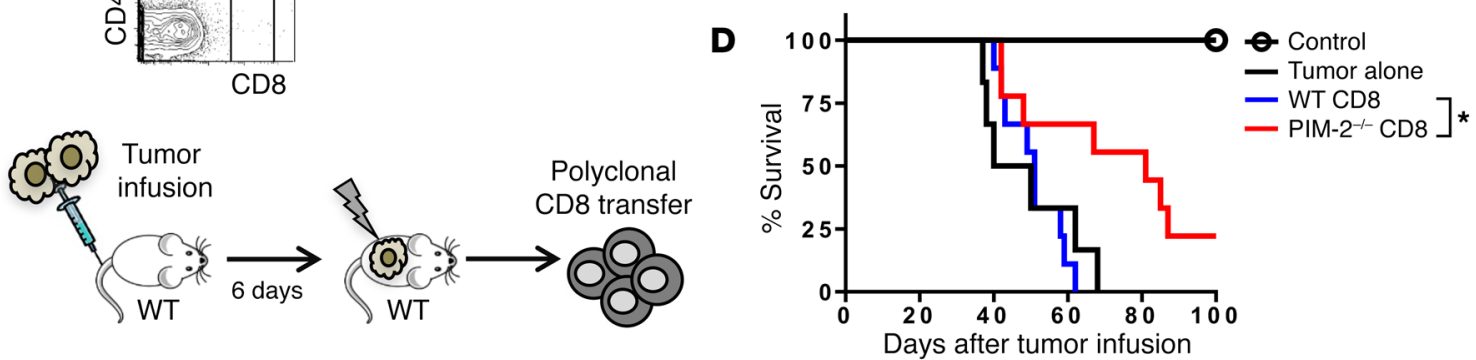

$\mathbf{E}$
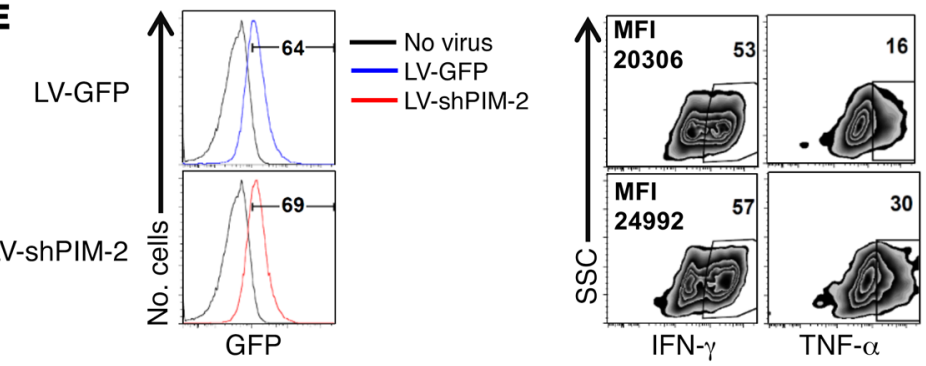

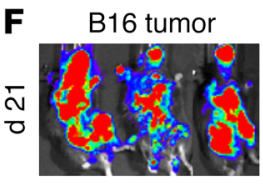

$\stackrel{\infty}{\sim}$

$\stackrel{\text { 色 }}{0}$
LV-GFP
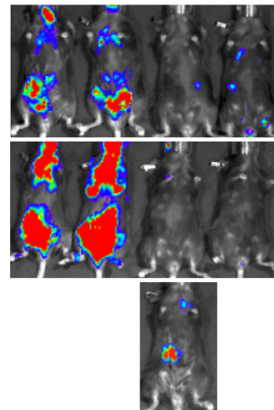

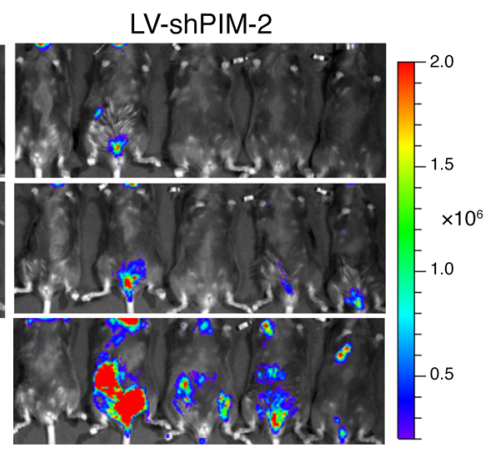

G

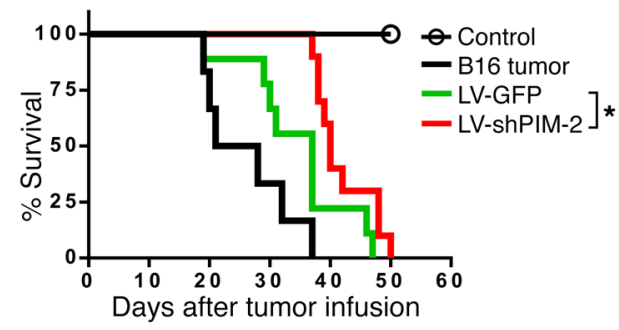

Figure 7. PIM-2 kinase suppresses antitumor immunity primarily mediated by CD8+ $\mathbf{T}$ cells. (A) Isotype control antibodies (IgC) or anti-CD8 (2.43) antibody were administered i.p. to PIM-2-1- tumor-bearing mice twice weekly ( $200 \mu \mathrm{g} / \mathrm{mouse})$. Depletion of CD8 ${ }^{+} \mathrm{T}$ cells was confirmed by flow cytometry before tumor infusion. (B) Survival of tumor-bearing mice treated with antibody was monitored until day 70 . WT tumor-bearing mice given IgG and heterozygous PIM-2 mice (PIM- $2^{+-}$) with no treatment were used as control groups ( $n=6$ mice per group). (C) Diagram of adoptive T cell transfer. $2 \times 10^{5}$ TS-1 tumor cells were infused into mice, and tumors were allowed to establish in WT mice for 6 days. Tumor-bearing mice were sublethally irradiated at 500 cGy followed by transfer of $2 \times 10^{6} \mathrm{CD}^{+} \mathrm{T}$ cells isolated from WT and PIM-2-/- mice. Irradiated tumor-bearing mice without T cell transfer were used as controls. (D) Survival of tumor-bearing mice is shown until day 100 ( $n=9$ mice per group). (E-G) B6 mice were injected i.v. with $5 \times 10^{5}$ B16 tumor cells and tumors that were allowed to establish for 6 days. These tumor-bearing mice were sublethally irradiated and received adoptive transfer of $1 \times 10^{6} \mathrm{Pmel}-1$ cells in which PIM-2 was silenced with LV-shPIM-2. (E) Virus transduction efficiency is illustrated by percentages of GFP+ cells. Transduced Pmel-1 cells were stimulated with PMA and ionomycin and measured for IFN- $\gamma$ and TNF- $\alpha$ secretion. (F) Tumor growth was monitored with bioluminescent imaging. (C) Survival of tumor-bearing mice is depicted ( $n=9-10$ mice per group) by log-rank test. ${ }^{*} P<0.05$, ${ }^{* * *} P<0.001$.

PIM-2-/- T cells compared with WT T cells (Supplemental Figure $8 \mathrm{~A})$. As p73 was also previously reported to regulate $\mathrm{T}$ cell apoptosis (19), we found that PIM-2 $2^{-/-}$T cells expressed decreased levels of p73 expression (Supplemental Figure 8B).
Activated $\mathrm{T}$ cells utilized glucose uptake and glycolysis for their energy (37). Hence, we hypothesized that PIM-2 $2^{-/-} \mathrm{T}$ cells would demonstrate increased glycolysis in response to alloantigen (38). To this end, we first sought to determine the level of phos- 
A

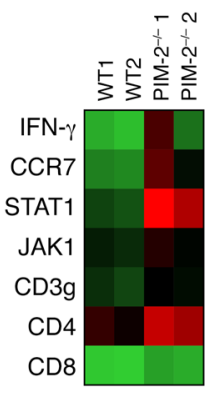

Upregulation

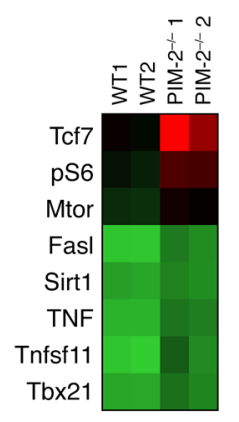

B

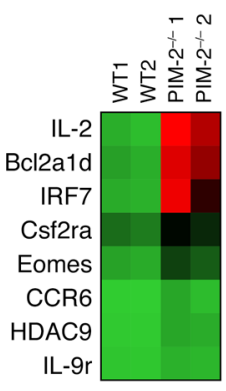

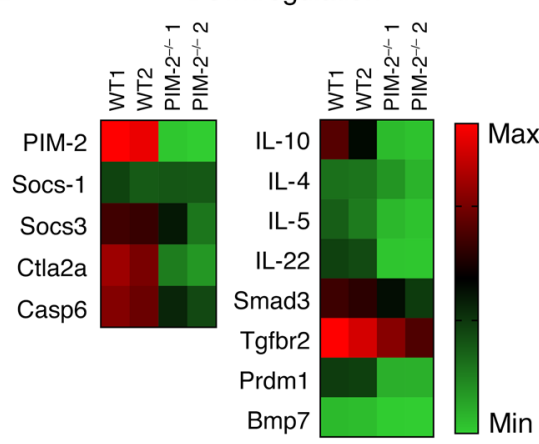
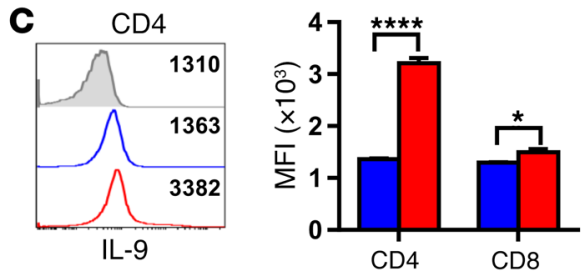

D

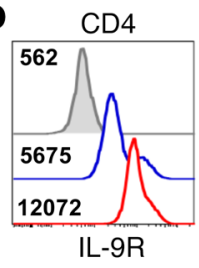

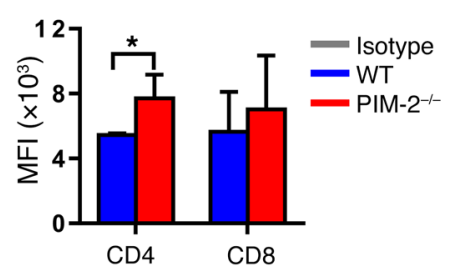

E

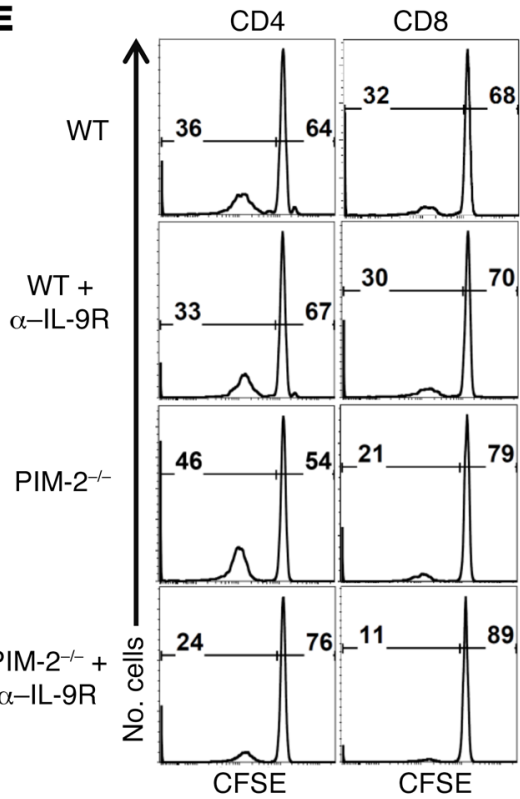

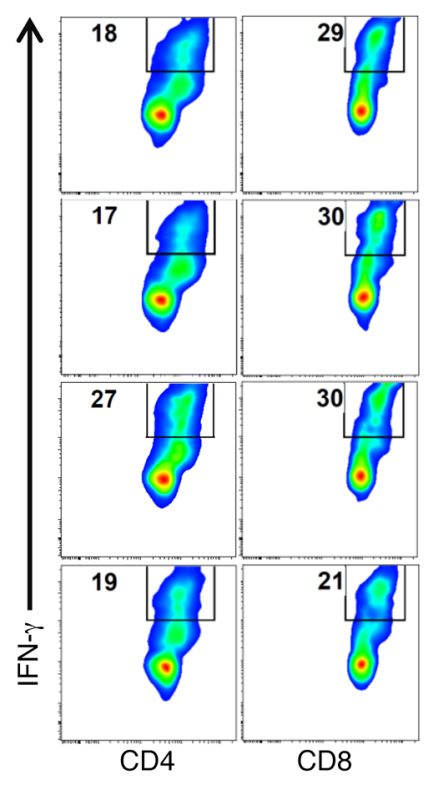

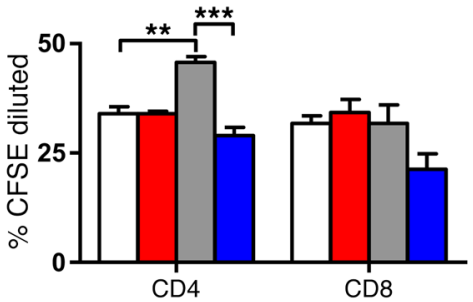

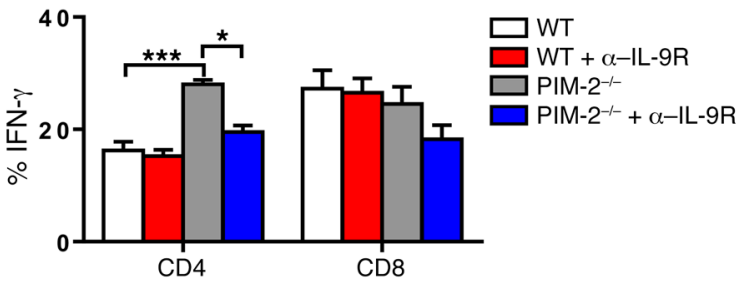

Figure 8. PIM-2 kinase regulates T cell alloresponses through IL-9/IL-9R signaling. (A and $\mathbf{B}$ ) Purified WT or PIM-2-- T cells were stimulated for 3 days with anti-CD3 and anti-CD28 ( $5 \mu \mathrm{g} / \mathrm{ml}$ each). The heatmap represents relative expression of RNA level; each graph represents maximum to minimum value of upregulation (A) or downregulation (B). (C) WT or

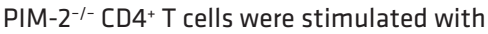
anti-CD3 and anti-CD28 $(2 \mu \mathrm{g} / \mathrm{ml}$ each) and polarized under Th9 polarizing conditions ( $10 \mathrm{ng} / \mathrm{ml} \mathrm{IL-} 4$ and $2 \mathrm{ng} / \mathrm{ml} \mathrm{TGF-} \beta$ ). The representative histogram plot shows mean fluorescence intensity (MFI) of IL-9, and quantified MFI is shown in the bar graph $(n=3)$. ( $\mathbf{D}$ and $\mathbf{E})$ Purified T cells of WT and PIM-2/- mice were cocultured with allogeneic antigen-presenting cells from B6 mice for 5 days in the presence or absence of anti-IL-9R. (D) IL-9R expression was measured on T cells $(n=4)$. (E) Cells were restimulated with PMA and ionomycin for IFN- $\gamma$ secretion. Percentages of CFSE-diluted and IFN- $\gamma$-producing cells on gated live donor $\mathrm{CD} 4^{+}$or $\mathrm{CD} 8^{+} \mathrm{T}$ cells $(n=4)$. Data represent mean \pm SEM by 2-tailed Student's $t$ test (C), MannWhitney test (D), and 1-way ANOVA and Tukey's HSD post hoc analysis (E). ${ }^{*} P<0.05,{ }^{* *} P<0.01,{ }^{* *} P<0.001$, $* * * * P<0.0001$. phorylated S6 (p-S6), a hallmark of mTOR activation. Indeed, p-S6 was significantly increased in PIM-2-/- CD $4^{+} \mathrm{T}$ cells (Supplemental Figure $8 \mathrm{C}$ ). These data were correlated with the higher production of IFN- $\gamma$ and GVHD severity induced by PIM-2-/- T cells. Hence, these transcriptional and metabolic changes are associated with increased antitumor immunity in PIM-2-/- T cells.

As we observed an increase of $I L-9 R$ RNA level, we hypothesized that the IL-9/IL-9R axis could confer enhanced antitumor immunity. Furthermore, Th9 and Tc 9 subsets have been increas- ingly shown to possess antitumor activity $(39,40)$. The IL-9R complex consists of IL-9R $\alpha$ and a common $\gamma\left(\gamma_{c}\right)$ chain, which is shared by other cytokine families such as IL-2, IL-4, IL-7, and IL-15. IL-9R was shown to signal through the phosphorylation of JAK1 and JAK3, resulting in the activation of STAT1, STAT3, and STAT5. The deficiency of IL-9R or IL-9 neutralization has previously been shown to attenuate inflammatory diseases $(41,42)$. We initially investigated Th9 and Tc9 differentiation of PIM-2-/- T cells under IL-9 polarizing conditions. Consistently, upregulation of IL-9R was associated 

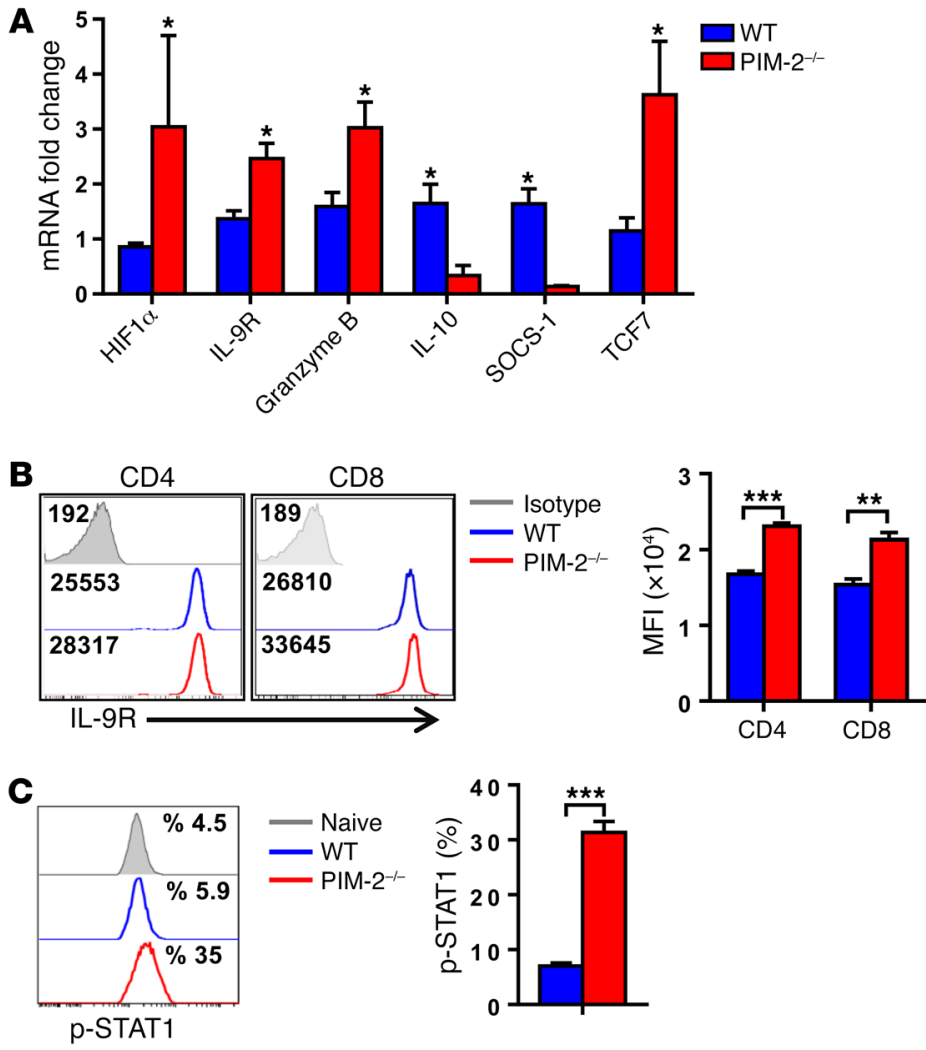

Figure 9. PIM-2-deficient T cells augment antitumor activity via IL-9R/STAT1 pathway. (A) Bar graphs show fold change of mRNA expression of HIF1 $\alpha, I L-9 R$, CZMB, IL-10, SOCS-1, and TCF7 on TILs evaluated by quantitative PCR ( $n=4$ mice per group). (B) Histogram analyses show IL-9R expression from TILs, and quantified MFI is shown in the bar graph. (C) Phospho-STAT1 was measured on T cells isolated from tumor sites and restimulated with IL-9 $(20 \mathrm{ng} / \mathrm{ml})$ in vitro. Percentages of $\mathrm{p}$-STAT1 are shown in the bar graph ( $n=3$ mice per group). Data represent mean \pm SEM by 2-tailed Student's $t$ test. ${ }^{*} P<0.05$, ${ }^{* *} P<0.01$, ${ }^{* * *} P<0.001$.

with an increase of Th9 and Tc 9 cell differentiation after polyclonal stimulation (Figure 8C). A significant increase of IL-9R expression has been confirmed in PIM-2-- ${ }^{--}$D $4^{+} \mathrm{T}$ cells after allogeneic stimulation (Figure 8D). Thus, we further investigated the function of IL-9 signaling in vitro and found that blocking of IL-9R is more pronounced in PIM-2- $2^{--}$cells as reflected by markedly reduced $\mathrm{T}$ cell proliferation and IFN- $\gamma$ production of $\mathrm{CD} 4^{+} \mathrm{T}$ cells (Figure $8 \mathrm{E}$ ).

PIM-2-deficient $T$ cells augment antitumor activity via $I L-9 R /$ STAT1 signaling. We then extended these studies in vivo by further examining TILs isolated from tumor-bearing mice. HIF1 $\alpha$ is a transcription factor that plays a key role in proinflammatory cytokine production. Loss of HIF1 $\alpha$ resulted in enhanced Treg differentiation and protected mice from autoimmune disease $(43,44)$. Although the previous study had shown that PIM-2 interacts with HIF1 $\alpha$ and enhances HIF $1 \alpha$ transcription under hypoxic conditions in HepG2 human liver cancer cells in vitro (45), we observed that PIM-2 $2^{-/} \mathrm{T}$ cells in tumors augmented HIF1 $\alpha$ mRNA activity. In addition, the TILs from tumor-bearing PIM- $2^{-/}$mice increased $I L-9 R$ and GZMB expression but decreased $I L-1 O$ and SOCS-1 mRNA levels (Figure 9A).

Similar induction of IL-9R expression was also observed in TILs isolated from PIM-2/- mice (Figure 9B). IL-9/IL-9R signal- ing has been shown to activate the STAT family. We observed an increased level of STAT1 mRNA, which could be due to stronger IL-9R signaling in PIM-2-- $\mathrm{T}$ cells. Indeed, we found that phospho-STAT1 (p-STAT1) was upregulated in PIM-2 ${ }^{--}$ $\mathrm{T}$ cells isolated from tumor sites after restimulation with recombinant IL-9 (Figure 9C). This result is also correlated with upregulation of FasL on TILs of PIM-2-/ T cells, which is in line with a previous study showing that activation of STAT1 was required for FasL expression (46). Collectively, our results have unraveled the role of the PIM-2 isoform, as distinguished from its family members, in $\mathrm{T}$ cells.

\section{Discussion}

Pan-PIM kinase inhibition in both tumor cells and $\mathrm{T}$ cells has been previously shown to reduce cell proliferation and survival $(10,47)$. The specific inhibition of PIM-2 also showed some promising results by delaying tumor burden in a multiple myeloma murine model (18). However, the effect of targeting PIM-2 kinase in primary $\mathrm{T}$ cells has not been previously investigated. In the current study, we observed that PIM-2-deficient $\mathrm{T}$ cells induced extremely severe GVHD after allo-BMT. Upon alloantigen activation, PIM-2-- $\mathrm{T}$ cells rapidly expanded and differentiated into the Th1 subset with a simultaneous upregulation of CXCR3 and $\alpha_{4} \beta_{7}$, whose elevated expression is known to promote $\mathrm{T}$ cell migration into GVHD target organs $(32,48)$. In contrast, PIM- $1 / 3^{---} \mathrm{T}$ cells demonstrated reduced $\mathrm{T}$ cell trafficking and less ability to proliferate as well as to produce cytokines. These physiological changes resulted in less severe GVHD than WT T cells. These results indicate that PIM-2 kinase negatively regulates $\mathrm{T}$ cells' responses to alloantigen and thus their pathogenicity to induce GVHD whereas PIM-1 and PIM-3 isoforms are positive regulators. Strikingly, $\mathrm{PIM}-2^{-/}$mice mediated robust rejection of syngeneic tumor primarily in a $\mathrm{CD}^{+} \mathrm{T}$ cell-dependent manner. Furthermore, this superior antitumor immunity in the absence of PIM-2 kinase is associated with upregulation of IL-9R expression, inflammatory cytokines, and FasL in conjunction with lower levels of infiltrated Tregs as well as PD-1 expression among TILs. These data indicate that PIM-2 expression suppresses $\mathrm{T}$ cell immunity against tumors.

$\mathrm{CD}^{+} \mathrm{T}$ cells play a critical role in mediating antitumor responses in PIM-2 $-{ }^{--}$mice as evidenced by the observation that depletion of $\mathrm{CD}^{+} \mathrm{T}$ cells abolished the antitumor effect in these mice. The $\mathrm{CD} 4^{+} \mathrm{T}$ cell subset may partially assist $\mathrm{CD} 8^{+} \mathrm{T}$ cells in tumor regression; however, adoptive transfer of polyclonal PIM-2 $2^{--} \mathrm{CD} 4^{+} \mathrm{T}$ cells alone or the combination of $\mathrm{PIM}-2^{-/-} \mathrm{CD} 4^{+}$ and $\mathrm{CD} 8^{+} \mathrm{T}$ cells into tumor-bearing mice did not protect mice from tumor relapse (Supplemental Figure 6). This result could be due to high plasticity of $\mathrm{CD} 4^{+} \mathrm{T}$ cells that may convert into iTregs in the tumor microenvironment (49). Nevertheless, adoptive transfer of polyclonal PIM-2- ${ }^{--} \mathrm{CD} 8^{+} \mathrm{T}$ cells and antigen-specific $\mathrm{CD} 8^{+} \mathrm{T}$ cells with silenced PIM- 2 could substantially delay tumor growth. Furthermore, we excluded the possibility that NK cells mediated antitumor responses, since NK cells are defective in $\mathrm{PIM}-2^{-/}$mice and the same tumor readily grew in $\mathrm{PIM}-2^{+/-}$mice.

In the current work, we primarily studied the properties of PIM-2 ${ }^{--} \mathrm{T}$ cells against syngeneic TS- 1 breast tumor; however, in 


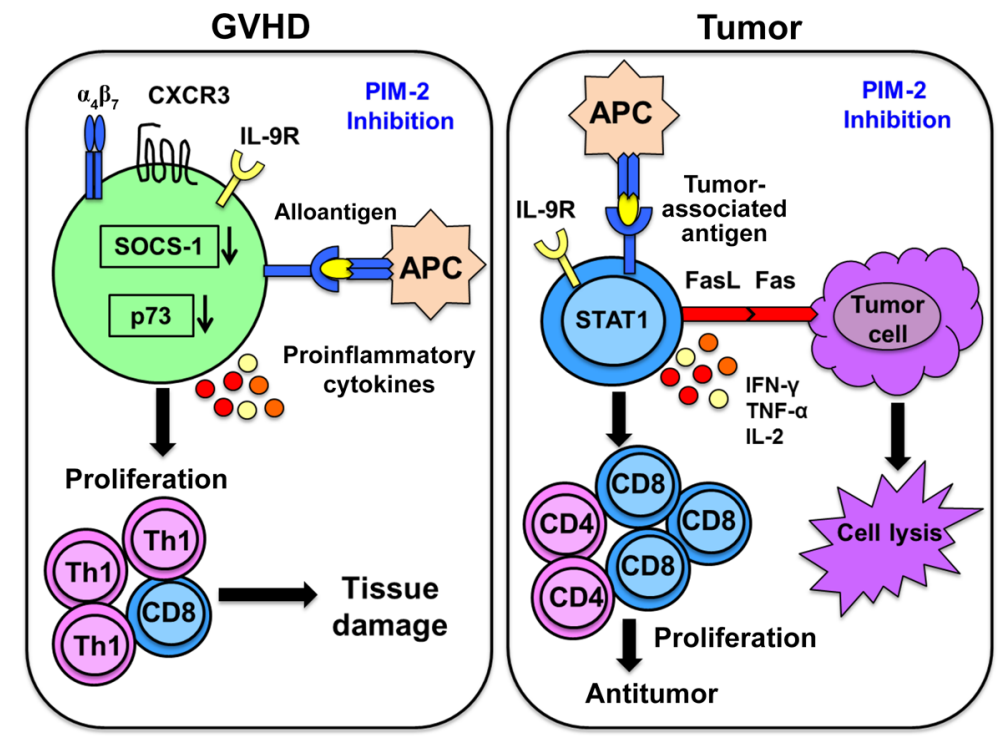

Figure 10. Inhibition of PIM-2 kinase in T cells induces severe GVHD while mediating cytolytic function against the tumor. The absence of PIM-2 isoform after allostimulation increases $\mathrm{T}$ cell proliferation, proinflammatory cytokines, and $\mathrm{CD} 4^{+} \mathrm{T}$ cell differentiation into a Th1 subset and causes tissue damage in a GVHD model. Despite the increased inflammatory responses, PIM-2-deficient T cells upregulate IL-9R, STAT1, and FasL expression and augment antitumor activity. APC, antigenpresenting cell. the clinic, autotransplant in patients with breast cancers has not been successful. Nevertheless, the non-immunogenicity of breast cancers is known, due to their heterogeneous mixture of different molecular subtypes with diversity in gene expression patterns (50). Tumor-infiltrating lymphocytes that recognize certain subsets of breast tumors are responsible for cancer therapy and are correlated with patient survival. For example, the $\mathrm{CD} 8^{+} \mathrm{T}$ cells that infiltrate into breast cancer tissue were associated with a decrease of breast cancer-specific mortality in the subcohort of ER-negative tumors and ER-positive/HER2-positive tumors (51). On the basis of our data, the immunogenicity of the TS- 1 tumor cell line is as low as that of human breast cancers, since the transfer of syngeneic WT $\mathrm{CD}^{+} \mathrm{T}$ cells had no effect in controlling tumor growth. However, PIM-2 $2^{-/-} \mathrm{T}$ cells could promote $\mathrm{T}$ cell responses against low-immunogenic tumors, which highlights the advantage of targeting PIM-2 kinase in T cells for cancer immunotherapy.

PIM-2 kinase was initially described as a regulator of cell apoptosis in different tumor cell lines and could maintain cell survival independent of PI3K and Akt pathways $(14,24)$. As a result, several studies have shown that PIM-2 kinase is a potential target for inhibiting cancer cell growth. Consistent with a previous study demonstrating that pan-PIM inhibitor blocked $\mathrm{T}$ cell acute lymphoblastic leukemia (T-ALL) growth and induced apoptotic cell death (52), we showed here that silencing PIM-2 kinase in murine T cell leukemia, T-ALL, and EL-4 (lymphoma cell line) sensitized tumor cells to apoptosis (Supplemental Figure 7, A and B). However, other studies showed that PIM-2 could have an opposite role under different conditions and thereby suppress cell survival and proliferation $(19,20)$.

Although PIM-2 was previously identified as promoting rapamycin-resistant $\mathrm{T}$ cell activation (21), it was also shown to prevent SOCS-1 from degradation and induce T cell anergy (23). Along the same lines, we observed a decreased level of SOCS-1 on activated PIM-2-/- T cells, supporting that PIM-2 kinase controls SOCS-1 stability. Moreover, this observation is in parallel with the in vivo finding that PIM-2 deficiency results in T cell hyperactivation and high levels of inflammatory cytokines in both GVHD and tumor models. Recently, PIM-2 kinase was shown to phosphory- late Foxp3 and negatively regulate Treg suppressive function and stability (22). However, these studies did not distinguish between the roles of PIM-2 kinase on natural Tregs (nTregs) and iTregs. The dextran sulfate sodium-induced colitis used in this study may not directly reflect the effect of PIM-2 kinase on Tregs. In contrast, we did not observe an effect of PIM-2 kinase on iTreg generation in a GVHD model (data not shown), but found that PIM-2 kinase promotes Treg infiltration to tumor sites and suppresses $\mathrm{T}$ cell response. Collectively, our data suggest that PIM-2 kinase clearly functions differently in malignant cells versus primary $\mathrm{T}$ cells.

PIM-2 kinase has been identified as interacting with substrates that are involved in apoptosis, cell cycle, metabolism, and cytokine signaling pathways. We observed that the molecular mechanisms underlying the augmented ability of PIM- $2^{--} \mathrm{T}$ cells to induce acute GVHD are a consequence of the downregulation of proapoptotic protein, p73, and SOCS-1. The robust Th1 polarization of PIM-2 ${ }^{-/-} \mathrm{T}$ cells after alloantigen stimulation enhanced $\mathrm{T}$ cell proliferation and migration to target organs and was responsible for lethality in mice. The deficiency of PIM-2 in T cells upregulated memory-associated genes such as TCF7, which was shown to promote long-term memory $T$ cells (53). In addition, these memoryassociated genes correlated with the level of Blimp-1. Prdm1 $1^{-/-} \mathrm{T}$ cells augmented the formation of memory-precursor cells (54). From our RNA sequencing data, we observed downregulation of $B L I M P-1$ in conjunction with upregulation of $I L-2$, which promotes T cell survival. PIM-2 kinase may regulate Blimp-1 through IL-2 repression and thereby suppress $\mathrm{T}$ cell functions (55).

Furthermore, our data demonstrate that PIM-2 kinase downregulated several cytolytic markers that are essential for cytotoxic $\mathrm{T}$ lymphocyte functions and served to further suppress T cell activity such as FasL, IFN- $\gamma$, and TNF- $\alpha$. A molecule that could potentially also be regulated by PIM-2 kinase and promote $\mathrm{T}$ cell function is IL-9R. We observed an upregulation of $I L-9 R$ in PIM-2-/- cells among the TILs; IL-9R is required for JAK/ STAT pathway activation (56). A recent study demonstrated that human melanoma-infiltrating $\mathrm{CD} 4^{+} \mathrm{CD} 8^{+}$double-positive $\mathrm{T}$ cells expressed a high level of IL-9R. IL-9/IL-9R signaling was dem- 
onstrated to enhance $\mathrm{T}$ cell survival, proliferation, and cytolytic activity through granzyme B (57). IL-9 is also known to inhibit cell apoptosis, and it was previously shown in mouse neurons that IL-9 reduced the expression of the proapoptotic molecule Bax (58). The increased level of IL-9R, STAT1 activation, and FasL expression in TILs of PIM-2-/- $\mathrm{T}$ cells may augment $\mathrm{T}$ cell survival and enhanced antitumor activity (Figure 10).

Adoptive T cell immunotherapy has been shown to successfully eradicate tumors. Several strategies have been developed to improve the efficacy of $\mathrm{T}$ cell immunotherapy - for example, selection of potent $\mathrm{T}$ cell subsets (53), cytokine administration to improve T cell survival (59), engineering of $\mathrm{T}$ cell receptors (chimeric antigen receptors) to specifically target cell surface molecules (60), and blocking of the immune checkpoint on T cells, such as PD-1, PD-L1, or CTLA-4, using monoclonal antibodies (61). Combinational strategies have also shown promising results in treating metastatic cancer (62). Our data provide evidence that targeting PIM-2 kinase has a therapeutic potential in combination with adoptive $T$ cell immunotherapy.

In summary, we have distinguished the unique activities of the PIM-2 kinase in T cells, which were opposed to the functions of its other family members, PIM-1 and PIM-3. These findings raise concerns that pan-PIM inhibition may have disadvantages in the context of suppressing the patient's immune responses and inducing $\mathrm{T}$ cell anergy. Because PIM-2 kinase negatively regulates $\mathrm{T}$ cell-mediated GVH and GVL responses, targeting of PIM-2 kinase may not be applicable in allogeneic hematopoietic cell transplantation (HCT), since it might exacerbate GVHD. In contrast, PIM-1 and PIM-3 isoforms may serve as potential therapeutic targets for alleviating GVHD. Importantly, our findings reveal a biological role of PIM-2 kinase in T cell immunity that had not been previously defined. They provide a strong rationale to target PIM-2 kinase for immunotherapy against hematologic malignancy with autologous HCT, as well as against solid tumors, particularly in adoptive $\mathrm{T}$ cell therapy.

\section{Methods}

Mice. B6 (B6, H-2 $)$, FVB (H-2 $)$, and BALB/c $\left(\mathrm{H}-2^{\mathrm{d}}\right)$ mice were purchased from the National Cancer Institute, NIH. The breeders of PIM mutant mice on an FVB background were kindly provided by Andrew S. Kraft (63). Animals were maintained in pathogen-free facilities in the American Association for Laboratory Animal Science-accredited Animal Resource Center at the Medical University of South Carolina. All mouse procedures were approved by the Institutional Animal Care and Use Committee of the Medical University of South Carolina.

$T$ cell purification. $T$ cells were purified from spleen and lymph nodes by negative selection with magnetically labeled biotin-conjugated antibodies against CD45R (clone RA3-B2), CD49b (clone DX5), CD11b (clone M1/70), and Ter-119 (clone Ter-119; all antibodies were from eBioscience) and anti-biotin microbeads (Miltenyi Biotec). $\mathrm{T}$ cells were depleted from BM (TCD-BM) using magnetically labeled CD4 (clone GK1.5, eBioscience) and CD8 (clone 53-6.7, eBioscience) biotin-conjugated antibodies.

GVHD and GVL models. Recipient female mice (8-10 weeks old) were lethally irradiated at $700 \mathrm{cGy}$ for BALB/c or 1,100 cGy for B6 and FVB (split dose) using an X-RAD 320 irradiator (Precision X-Ray Inc.). Syngeneic (FVB) or allogeneic (BALB/c) irradiated mice underwent transplantation with $5 \times 10^{6} \mathrm{TCD}-\mathrm{BM}$ per mouse plus various numbers of T cells from FVB donors as indicated. Recipient survival and body weight were monitored for 80 days. For the GVL model, Luc/ neo-transduced A2O B cell lymphoma (ATCC) was infused on the day of BMT ( $2 \times 10^{3} \mathrm{~A} 20$ per mouse). Tumor growth was measured with bioluminescent imaging (64) using a Xenogen-IVIS 200 in vivo Imaging System (PerkinElmer). The MLL-AF9 tumor cells were provided by Sophie Paczesny (Indiana University School of Medicine, Indianapolis, Indiana, USA) (34); $2 \times 10^{4}$ cells per mouse were used, and percentages of $\mathrm{CD} 11 \mathrm{~b}^{+} \mathrm{GFP}^{+}$in peripheral blood were analyzed by flow cytometry. Recipient survival was monitored until day 50 .

Tumor lung model and adoptive T cell transfer. The TS-1-TGL cell line, PyMT mammary tumor, was provided by Johanna A. Joyce (Memorial Sloan Kettering Cancer Center, New York, New York, USA). TS-1 (H-2 was established by i.v. injection $\left(2 \times 10^{5}\right.$ per mouse) in the lung model and adoptive transfer model or by s.c. injection $\left(5 \times 10^{5}\right.$ per mouse). B16-F10-fluc (ATCC) expressing luciferase $\left(5 \times 10^{5}\right)$ mouse melanoma tumor was used in B6 mice. After 6 days of tumor establishment, recipient mice were sublethally irradiated with 500 cGy. Tumor-bearing mice were adoptively transferred with either $2 \times 10^{6}$ per mouse purified CD $8^{+}$ T cells from FVB mice or $1 \times 10^{6}$ per mouse CD $8^{+}$Pmel- 1 T cells on day 7. Tumor relapse was monitored until endpoints. For TIL isolation from lung, the tissue was cut into small pieces and digested with collagenase type IV (0.5 mg/ml; Sigma-Aldrich) and DNase I (Worthington) for 90 minutes at $37^{\circ} \mathrm{C}$. The TILs were later enriched by density gradient centrifugation on 40/80 Percoll gradients (GE Healthcare).

Antibodies and flow cytometry. The following antibodies were used for cell surface staining: anti-CD4 (clone RM4-5, BD Biosciences), anti-CD8 (clone 53-6.7, BD Biosciences), anti-H-2 ${ }^{\mathrm{q}}$ (KH114, Biolegend), anti-CXCR3-biotin (CXCR3-173, eBioscience), anti- $\alpha_{4} \beta_{7}$ (DATK32, BD Biosciences), anti-FasL (MFL3, BD Biosciences), antiPD-1 (MFL3, eBioscience), anti-NK1.1 (PK136, eBioscience), antiCD44 (IM7, Biolegend), anti-CD62L (MEL-14, eBioscience), antiTCR $\beta$ (H57-597, BD Biosciences), anti-CD11b (M1/70, eBioscience), and anti-IL-9R (R\&D Systems, clone 224325). To measure intracellular cytokines, cells were stimulated for $4-5$ hours at $37^{\circ} \mathrm{C}$ with PMA (100 ng/ml; Sigma-Aldrich) and ionomycin (100 ng/ml; Calbiochem, Millipore) in the presence of GolgiStop (BD Biosciences). Fixation and permeabilization were performed using Cytofix/Cytoperm Plus (BD Biosciences), followed by staining with the appropriate antibodies, including anti-IFN- $\gamma$ (clone XMG1.2, eBioscience), anti-IL-17 (clone TC11-18H10.1, BioLegend), anti-IL-4 (clone 11B11, BD Biosciences), anti-IL-5 (clone TRFK5, eBioscience), anti-Foxp3 (clone FJK16s, eBioscience), anti-IL-9 (RM9A4, Biolegend), anti-TNF- $\alpha$ (MP6XT22, BD Biosciences), anti-IL-2 (554428, BD Biosciences), anti-Ki67 (16A8, Biolegend), anti-STAT1 (pY701, BD Biosciences), and antipS6-AF467 (clone D57.2.2E, Cell Signaling Technology). LIVE/DEAD yellow cell staining kit (catalog L-34968) and CFSE (catalog C1157) were purchased from Invitrogen.

Western blotting. Whole-cell lysates were collected using lysis buffer supplemented with the protease inhibitor cocktail solution according to the manufacturer's protocol (MCL-1, Sigma-Aldrich). Cell lysates were electrophoresed in precast polyacrylamide gel and blotted onto PVDF membranes (Bio-Rad Laboratories Inc.). After blocking with $5 \%$ nonfat dry milk, the membranes were incubated with primary antibodies overnight at $4^{\circ} \mathrm{C}$, followed by washing and addition of a horseradish peroxidase-conjugated secondary antibody 
for 1 hour. All antibodies were from Santa Cruz Biotechnology: PIM-2 (1D12), SOCS-1 (H93), p73 (H79). Monoclonal anti- $\beta$-actin antibody (AC-74) was purchased from Sigma-Aldrich. Blots were treated with ECL Western Blotting Substrate (Thermo Fisher Scientific) and visualized by exposure to photographic film.

Lentiviral transduction. Lentiviral vectors of mouse PIM-2 (mGFPtagged, MR222342L2) and shRNA-PIM-2 (24) were generated and purchased from Origene Inc. Lentiviral particles were produced by 293 T cells (ATCC) according to standard protocols $(65,66)$. Purified T cells were activated for 48 hours in a plate coated with $5 \mu \mathrm{g} / \mathrm{ml}$ antiCD3 (145-2C11, Bio X Cell) and $5 \mu \mathrm{g} / \mathrm{ml}$ anti-CD28 (37.51, Bio X Cell). Lentivirus with $\mathrm{T}$ cells was spin-infected at $32^{\circ} \mathrm{C}$ for 2 hours in the presence of $5 \mu \mathrm{g} / \mathrm{ml}$ protamine sulfates (P2162, Sigma-Aldrich). After overnight transduction, cells were washed and checked for transduction efficiency with flow cytometry (GFP) or Western blot analyses before being used for experiments.

RNA isolation, reverse transcription, and quantitative PCR. Total RNA was extracted from TILs using Trizol (Invitrogen). cDNA was generated from $1 \mu \mathrm{g}$ total RNA using iScript cDNA Synthesis Kit (BioRad Laboratories Inc.). SYBR Green incorporation of quantitative PCR was performed using a SYBR Green mix in the CFX96 Detection System (Bio-Rad Laboratories Inc.). Samples from each experimental condition were run in duplicates and were normalized to $\beta$-actin. Primer sequences were obtained from Primer Bank.

Statistics. For comparison of recipient survival and tumor mortality rate in all experiments, the log-rank (Mantel-Cox) test (GraphPad Prism software, version 7) was used to determine statistical significance. To compare cell frequency and cytokine and cytolytic marker expression, a 2-tailed Student's $t$ test or a nonparametric MannWhitney test was performed to determine any statistical differences between 2 groups. Based on graphical evaluation of distributions of flow cytometry endpoints using histograms and quantile-quantile plots, data were log-transformed to induce approximate normality and stabilize variance across groups before data analysis. Group means were then estimated using 1-way ANOVA, and Tukey's honest significant difference (HSD) post hoc analysis was used to adjust $P$ values for multiple pairwise comparisons between conditions. A $P$ value less than 0.05 was considered to be statistically significant.
Study approval. All mice were housed in a pathogen-free facility at the American Association for Laboratory Animal Science-accredited Animal Resource Center at the Medical University of South Carolina. All animal studies were performed under protocols approved by the Institutional Animal Care and Use Committee at the Medical University of South Carolina.

\section{Author contributions}

$\mathrm{AD}$ participated in experimental design, performed experiments, analyzed and interpreted data, and wrote the manuscript. YW participated in experimental design and performed a part of a study for data collection and analysis. SI, HDN, DB, MZ, MHS, and SC participated in conducting experiments and acquiring data. ASK provided genetic knockout mice. EGH provided statistical analysis. SM and ASK participated in experimental design and revising the manuscript. XZY designed research, interpreted data, and edited and revised the manuscript.

\section{Acknowledgments}

We thank the flow cytometry core, a small-animal imaging core, at the Medical University of South Carolina for their assistance. We thank Ryan Sprissler (University of Arizona, Tucson, Arizona, USA) for RNA sequencing results and analysis. We thank Johanna A. Joyce for the TS-1 cell line and Sophie Paczesny for MLL-AF9 tumor cells. This work was supported in part by the Biostatistics Shared Resource, Hollings Cancer Center, Medical University of South Carolina (P30 CA138313). A.S. Kraft was supported by P30 CA023074 (University of Arizona Cancer Center), NIH R01CA173200, and DOD W81XWH-12-1-0560. This work is partially supported by NIH grants R01 CA169116, R01 AI118305, R01 HL137373, and R21 CA192202 and the SmartState Endowment in Cancer Stem Cell Biology \& Therapy Program to XZY.

Address correspondence to: Xue-Zhong Yu, Department of Microbiology and Immunology, HCC350, MSC955, Medical University of South Carolina, 86 Jonathan Lucas Street, Charleston, South Carolina 29425-5090, USA. Phone: 843.792.4756; Email: yux@musc.edu.
1. Warfel NA, Kraft AS. PIM kinase (and Akt) biology and signaling in tumors. Pharmacol Ther. 2015;151:41-49.

2. Mondello P, Cuzzocrea S, Mian M. Pim kinases in hematological malignancies: where are we now and where are we going? J Hematol Oncol. 2014;7:95.

3. Baytel D, Shalom S, Madgar I, Weissenberg R, Don J. The human Pim-2 proto-oncogene and its testicular expression. Biochim Biophys Acta. 1998;1442(2-3):274-285.

4. Narlik-Grassow M, Blanco-Aparicio C, Carnero A. The PIM family of serine/threonine kinases in cancer. Med Res Rev. 2014;34(1):136-159.

5. Gómez-Abad C, et al. PIM2 inhibition as a rational therapeutic approach in B-cell lymphoma. Blood. 2011;118(20):5517-5527.

6. Lu J, et al. Pim2 is required for maintaining multiple myeloma cell growth through modulating TSC2 phosphorylation. Blood.
2013;122(9):1610-1620.

7. Ren K, et al. The over-expression of Pim-2 promote the tumorigenesis of prostatic carcinoma through phosphorylating eIF4B. Prostate. 2013;73(13):1462-1469.

8. Chen WW, Chan DC, Donald C, Lilly MB, Kraft AS. Pim family kinases enhance tumor growth of prostate cancer cells. Mol Cancer Res. 2005;3(8):443-451.

9. Bullock AN, et al. Crystal structure of the PIM2 kinase in complex with an organoruthenium inhibitor. PLoS One. 2009;4(10):e7112.

10. Keeton EK, et al. AZD1208, a potent and selective pan-Pim kinase inhibitor, demonstrates efficacy in preclinical models of acute myeloid leukemia. Blood. 2014;123(6):905-913.

11. Xia Z, et al. Synthesis and evaluation of novel inhibitors of Pim-1 and Pim-2 protein kinases. JMed Chem. 2009;52(1):74-86.

12. Beharry Z, et al. Novel benzylidene-thiazoli- dine-2,4-diones inhibit Pim protein kinase activity and induce cell cycle arrest in leukemia and prostate cancer cells. Mol Cancer Ther. 2009;8(6):1473-1483.

13. Sakai I, Kraft AS. The kinase domain of Jak2 mediates induction of bcl-2 and delays cell death in hematopoietic cells. J Biol Chem. 1997;272(19):12350-12358.

14. Yan B, et al. The PIM-2 kinase phosphorylates $\mathrm{BAD}$ on serine 112 and reverses BAD-induced cell death. J Biol Chem. 2003;278(46):45358-45367.

15. Yu Z, et al. Proviral insertion in murine lymphomas 2 (PIM2) oncogene phosphorylates pyruvate kinase M2 (PKM2) and promotes glycolysis in cancer cells. J Biol Chem. 2013;288(49):35406-35416.

16. Zhang XH, Yu HL, Wang FJ, Han YL, Yang WL. Pim-2 modulates aerobic glycolysis and energy production during the development of colorectal tumors. Int JMed Sci. 2015;12(6):487-493.

17. Keane NA, Reidy M, Natoni A, Raab MS, 
O'Dwyer M. Targeting the Pim kinases in multiple myeloma. Blood Cancer J. 2015;5:e325.

18. Nair JR, et al. Novel inhibition of PIM2 kinase has significant anti-tumor efficacy in multiple myeloma. Leukemia. 2017;31(8):1715-1726.

19. Levy D, et al. Activation of cell cycle arrest and apoptosis by the proto-oncogene Pim-2. PLoS One. 2012;7(4):e34736.

20. Wang Z, Zhang Y, Gu JJ, Davitt C, Reeves R, Magnuson NS. Pim-2 phosphorylation of p21(Cip1/ WAF1) enhances its stability and inhibits cell proliferation in HCT116 cells. Int J Biochem Cell Biol. 2010;42(6):1030-1038.

21. Fox CJ, Hammerman PS, Thompson CB. The Pim kinases control rapamycin-resistant $\mathrm{T}$ cell survival and activation. J Exp Med. 2005;201(2):259-266.

22. Deng G, et al. Pim-2 kinase influences regulatory $T$ cell function and stability by mediating Foxp3 protein N-terminal phosphorylation. J Biol Chem. 2015;290(33):20211-20220.

23. Chen XP, et al. Pim serine/threonine kinases regulate the stability of Socs-1 protein. Proc Natl Acad Sci U S A. 2002;99(4):2175-2180.

24. Fox CJ, Hammerman PS, Cinalli RM, Master SR, Chodosh LA, Thompson CB. The serine/ threonine kinase Pim-2 is a transcriptionally regulated apoptotic inhibitor. Genes Dev. 2003;17(15):1841-1854.

25. Morishita D, Katayama R, Sekimizu K, Tsuruo T, Fujita N. Pim kinases promote cell cycle progression by phosphorylating and down-regulating p27Kip1 at the transcriptional and posttranscriptional levels. Cancer Res. 2008;68(13):5076-5085.

26. Song JH, Kraft AS. Pim kinase inhibitors sensitize prostate cancer cells to apoptosis triggered by Bcl-2 family inhibitor ABT-737. Cancer Res. 2012;72(1):294-303.

27. Albert MH, Yu XZ, Martin PJ, Anasetti C. Prevention of lethal acute GVHD with an agonistic CD28 antibody and rapamycin. Blood. 2005;105(3):1355-1361.

28. Mikkers H, et al. Mice deficient for all PIM kinases display reduced body size and impaired responses to hematopoietic growth factors. $\mathrm{Mol}$ Cell Biol. 2004;24(13):6104-6115.

29. Anderson BE, et al. Memory CD4+ T cells do not induce graft-versus-host disease. JClin Invest. 2003;112(1):101-108.

30. Dutt $\mathrm{S}$, et al. Naive and memory T cells induce different types of graft-versus-host disease. JImmunol. 2007;179(10):6547-6554.

31. Wysocki CA, Panoskaltsis-Mortari A, Blazar BR, Serody JS. Leukocyte migration and graft-versushost disease. Blood. 2005;105(11):4191-4199.

32. Waldman $E$, et al. Absence of $\beta 7$ integrin results in less graft-versus-host disease because of decreased homing of alloreactive $\mathrm{T}$ cells to intestine. Blood. 2006;107(4):1703-1711.

33. Kappel LW, et al. IL-17 contributes to CD4mediated graft-versus-host disease. Blood. 2009;113(4):945-952.

34. Zhang J, et al. ST2 blockade reduces SST2- producing $\mathrm{T}$ cells while maintaining protective mST2-expressing $\mathrm{T}$ cells during graft-versus-host disease. Sci Transl Med. 2015;7(308):308ra160.

35. Shree T, et al. Macrophages and cathepsin proteases blunt chemotherapeutic response in breast cancer. Genes Dev. 2011;25(23):2465-2479.

36. Caldwell SA, Ryan MH, McDuffie E, Abrams SI. The Fas/Fas ligand pathway is important for optimal tumor regression in a mouse model of CTL adoptive immunotherapy of experimental CMS4 lung metastases. JImmunol. 2003;171(5):2402-2412.

37. Nguyen HD, et al. Metabolic reprogramming of alloantigen-activated $\mathrm{T}$ cells after hematopoietic cell transplantation. JClin Invest. 2016;126(4):1337-1352.

38. Song JH, et al. Deletion of Pim kinases elevates the cellular levels of reactive oxygen species and sensitizes to K-Ras-induced cell killing. Oncogene. 2015;34(28):3728-3736.

39. Lu Y, et al. Th9 cells promote antitumor immune responses in vivo. J Clin Invest. 2012;122(11):4160-4171.

40. Lu Y, et al. Tumor-specific IL-9-producing CD8+ Tc9 cells are superior effector than type-I cytotoxic Tc1 cells for adoptive immunotherapy of cancers. Proc Natl Acad Sci U S A. 2014;111(6):2265-2270.

41. Li H, Nourbakhsh B, Ciric B, Zhang GX, Rostami A. Neutralization of IL- 9 ameliorates experimental autoimmune encephalomyelitis by decreasing the effector $\mathrm{T}$ cell population. JImmunol. 2010;185(7):4095-4100.

42. Nowak EC, et al. IL-9 as a mediator of Th17driven inflammatory disease. JExp Med. 2009;206(8):1653-1660.

43. Dang EV, et al. Control of T(H)17/T(reg) balance by hypoxia-inducible factor 1 . Cell. 2011;146(5):772-784.

44. Shi LZ, et al. HIF1alpha-dependent glycolytic pathway orchestrates a metabolic checkpoint for the differentiation of TH17 and Treg cells. JExp Med. 2011;208(7):1367-1376.

45. Yu Z, et al. A regulatory feedback loop between HIF-1 $\alpha$ and PIM2 in HepG2 cells. PLoS One. 2014;9(2):e88301.

46. Xu X, Fu XY, Plate J, Chong AS. IFN-gamma induces cell growth inhibition by Fas-mediated apoptosis: requirement of STAT1 protein for upregulation of Fas and FasL expression. Cancer Res. 1998;58(13):2832-2837.

47. Jackson LJ, et al. The role of PIM kinases in human and mouse $\mathrm{CD} 4+\mathrm{T}$ cell activation and inflammatory bowel disease. Cell Immunol. 2012;272(2):200-213.

48. Duffner U, et al. Role of CXCR3-induced donor T-cell migration in acute GVHD. Exp Hematol. 2003;31(10):897-902.

49. Zhou L, Chong MM, Littman DR. Plasticity of $\mathrm{CD} 4^{+} \mathrm{T}$ cell lineage differentiation. Immunity. 2009;30(5):646-655.

50. Perou CM, et al. Molecular portraits of human breast tumours. Nature. 2000;406(6797):747-752.

51. Ali HR, et al. Association between $\mathrm{CD} 8^{+} \mathrm{T}$-cell infiltration and breast cancer survival in 12,439 patients. Ann Oncol. 2014;25(8):1536-1543.

52. Padi SKR, et al. Targeting the PIM protein kinases for the treatment of a T-cell acute lymphoblastic leukemia subset. Oncotarget. 2017;8(18):30199-30216

53. Muranski P, et al. Th17 cells are long lived and retain a stem cell-like molecular signature. Immunity. 2011;35(6):972-985.

54. Rutishauser RL, et al. Transcriptional repressor Blimp-1 promotes CD8(+) T cell terminal differentiation and represses the acquisition of central memory $\mathrm{T}$ cell properties. Immunity. 2009;31(2):296-308.

55. Martins GA, Cimmino L, Liao J, Magnusdottir E, Calame K. Blimp-1 directly represses Il2 and the Il2 activator Fos, attenuating T cell proliferation and survival. J Exp Med.2008;205(9):1959-1965.

56. Demoulin JB, et al. A single tyrosine of the interleukin-9 (IL-9) receptor is required for STAT activation, antiapoptotic activity, and growth regulation by IL-9. Mol Cell Biol. 1996;16(9):4710-4716.

57. Parrot T, et al. IL-9 promotes the survival and function of human melanoma-infiltrating CD4(+) CD8(+) double-positive T cells. Eur J Immunol. 2016;46(7):1770-1782.

58. Fontaine RH, et al. IL-9/IL-9 receptor signaling selectively protects cortical neurons against developmental apoptosis. Cell Death Differ. 2008;15(10):1542-1552.

59. Zhang L, et al. Tumor-infiltrating lymphocytes genetically engineered with an inducible gene encoding interleukin-12 for the immunotherapy of metastatic melanoma. Clin Cancer Res. 2015;21(10):2278-2288.

60. Barrett DM, Singh N, Porter DL, Grupp SA, June $\mathrm{CH}$. Chimeric antigen receptor therapy for cancer. Annu Rev Med. 2014;65:333-347.

61. Chen L, Han X. Anti-PD-1/PD-L1 therapy of human cancer: past, present, and future. J Clin Invest. 2015;125(9):3384-3391.

62. Melero I, Berman DM, Aznar MA, Korman AJ, Pérez Gracia JL, Haanen J. Evolving synergistic combinations of targeted immunotherapies to combat cancer. Nat Rev Cancer. 2015;15(8):457-472.

63. An N, et al. Pim1 serine/threonine kinase regulates the number and functions of murine hematopoietic stem cells. Stem Cells. 2013;31(6):1202-1212.

64. Heinrichs J, et al. CD8(+) Tregs promote GVHD prevention and overcome the impaired GVL effect mediated by CD4(+) Tregs in mice. Oncoimmunology. 2016;5(6):e1146842.

65. Stripecke R. Lentiviral vector-mediated genetic programming of mouse and human dendritic cells. Methods Mol Biol. 2009;506:139-158.

66. Daenthanasanmak A, et al. Integrase-defective lentiviral vectors encoding cytokines induce differentiation of human dendritic cells and stimulate multivalent immune responses in vitro and in vivo. Vaccine. 2012;30(34):5118-5131. 